\title{
DISCRETE TIME MARKOVIAN AGENTS INTERACTING THROUGH A POTENTIAL
}

\author{
Amarjit Budhiraja ${ }^{1}$, Pierre Del Moral ${ }^{2}$ and Sylvain Rubenthaler ${ }^{3}$
}

\begin{abstract}
A discrete time stochastic model for a multiagent system given in terms of a large collection of interacting Markov chains is studied. The evolution of the interacting particles is described through a time inhomogeneous transition probability kernel that depends on the 'gradient' of the potential field. The particles, in turn, dynamically modify the potential field through their cumulative input. Interacting Markov processes of the above form have been suggested as models for active biological transport in response to external stimulus such as a chemical gradient. One of the basic mathematical challenges is to develop a general theory of stability for such interacting Markovian systems and for the corresponding nonlinear Markov processes that arise in the large agent limit. Such a theory would be key to a mathematical understanding of the interactive structure formation that results from the complex feedback between the agents and the potential field. It will also be a crucial ingredient in developing simulation schemes that are faithful to the underlying model over long periods of time. The goal of this work is to study qualitative properties of the above stochastic system as the number of particles $(N)$ and the time parameter $(n)$ approach infinity. In this regard asymptotic properties of a deterministic nonlinear dynamical system, that arises in the propagation of chaos limit of the stochastic model, play a key role. We show that under suitable conditions this dynamical system has a unique fixed point. This result allows us to study stability properties of the underlying stochastic model. We show that as $N \rightarrow \infty$, the stochastic system is well approximated by the dynamical system, uniformly over time. As a consequence, for an arbitrarily initialized system, as $N \rightarrow \infty$ and $n \rightarrow \infty$, the potential field and the empirical measure of the interacting particles are shown to converge to the unique fixed point of the dynamical system. In general, simulation of such interacting Markovian systems is a computationally daunting task. We propose a particle based approximation for the dynamic potential field which allows for a numerically tractable simulation scheme. It is shown that this simulation scheme well approximates the true physical system, uniformly over an infinite time horizon.
\end{abstract}

Mathematics Subject Classification. 60J05, 60K35, 92C45, 70K20, 60K40.

Received July 13, 2011. Revised May 23, 2012.

\footnotetext{
Keywords and phrases. Interacting Markov chains, agent based modeling, multi-agent systems, propagation of chaos, non-linear Markov processes, stochastic algorithms, stability, particle approximations, swarm simulations, chemotaxis, reinforced random walk.

1 Department of Statistics and Operations Research, University of North Carolina, Chapel Hill, NC, USA.

budhiraja@email.unc.edu

2 INRIA Bordeaux-Sud Ouest, Bordeaux Mathematical Institute, Université Bordeaux I, 351 cours de la Libération, 33405 Talence Cedex, France. Pierre.Del_Moral@inria.fr

3 Laboratoire de Mathématiques J.A. Dieudonné, Université de Nice-Sophia Antipolis, Parc Valrose, 06108 Nice Cedex 02, France. rubentha@unice.fr
} 


\section{INTRODUCTION}

In recent years there has been a significant interest in agent based modeling for complex systems. Examples of such models abound in physical and social sciences and include problems of biological aggregation [19], chemotactic response dynamics [11], self organized networks [16], communication systems [14], opinion dynamics [15], multi-target tracking [4], etc. See [14,18] for a comprehensive list of references. One popular model for interacting multi-agent systems (see [18] and references therein) consists of a large number of particles that influence each other indirectly through a dynamic potential field and can formally be described through the following system of equations.

$$
\begin{gathered}
\mathrm{d} X_{i}(t)=\nabla h\left(X_{i}(t), t\right) \mathrm{d} t+\mathrm{d} W_{i}(t), X_{i}(0)=x_{i} \in \mathbb{R}^{d}, i=1, \ldots, N . \\
\frac{\partial}{\partial t} h(t, x)=-\alpha h(t, x)+D \Delta h(t, x)+\beta \sum_{i=1}^{N} g\left(X_{i}(t), x\right), \quad h(0, x)=h_{0}(x) .
\end{gathered}
$$

Here $W_{i}, i=1 \ldots, N$ are independent Brownian motions which drive the state processes $X_{i}$ of the $N$ interacting particles. The interaction between the particles arises indirectly through the underlying potential field $h$ which changes continuously according to a diffusion equation and through the aggregated input of the $N$ particles. One example of such an interaction is in chemotactic cell response dynamics where cells preferentially move towards a higher chemical concentration and themselves release chemicals into the medium, in response to the local information on the environment, thus modifying the potential field dynamically over time. In this context, $h(t, x)$ represents the concentration of a chemical at time $t$ and location $x$. Diffusion of the chemical in the medium is captured by the Laplacian in (1.1) and the constant $\alpha>0$ models the rate of decay or dissipation of the chemical. Contribution of the agents to the chemical concentration field is given through the last term in the equation. The function $g$ captures the agent response rules and can be used to model a wide range of phenomenon [18]. The first equation in (1.1) describes the motion of a particle in terms of a diffusion process with drift in the direction of the gradient of the chemical concentration. Many variations of this basic model with applications to a wide range of fields can be found in [18]. A precise mathematical treatment of (1.1) presents significant technical obstacles and existing results in literature are limited to simulation and formal asymptotic approximations of the system. In the current work we will study a discrete time model which captures essential features of the dynamics in (1.1) and is also amenable to a rigourous mathematical treatment. The time evolution of the agents will be described through a time inhomogeneous transition probability kernel, where the kernel at time instant $n$ is determined in terms of the "gradient of the potential field" at time instant $n-1$. The agents in turn affect and contribute to the potential field dynamically over time. Thus as in the formal continuous time setting described above, the $N$-agent dynamics is strongly coupled and describes a (time inhomogeneous) Markov chain in $E^{N}$ where $E$ (a compact subset of $\mathbb{R}^{d}$ ) is the state space of a typical agent. Although the model description is considerably simpler in discrete time, our objective here is to go beyond formal heuristics (as is the current state of the art for the continuous time model in (1.1)) and to formulate and study precise mathematical properties of the system. We will establish convergence of the stochastic model to the solution of a non-linear dynamical system, over an arbitrary fixed time horizon, as the number of agents approach infinity and as a consequence obtain a propagation of chaos result (Thm. 1.2 and Cor. 1.3). We are particularly interested in the stability of the system as $N \rightarrow \infty$, over long periods of time. A mathematical understanding of the stability behaviour is key to the study of long term structure formation resulting from the complex interactions between the agents and the potential field. Stability results for the system are also crucial ingredients for studying the behaviour of approximate simulation schemes over long intervals of time. Denoting by $n$ the time parameter, we will give conditions under which, as $N \rightarrow \infty$ and $n \rightarrow \infty$ (in any order) the potential field and the empirical measure of the $N$ particles converges to limits that are independent of the initial configuration (Cor. 1.10). These limits are characterized as the unique fixed point of the limit deterministic non-linear dynamical system (Thm. 1.6). Uniform in time convergence of the stochastic model to the non-linear deterministic dynamics is established as well (Thm. 1.9). In general, simulation of interacting Markovian systems is a computationally 
daunting task. We propose a particle based approximation for the dynamic potential field which allows for a numerically tractable simulation scheme. Using the above stability results we show that this simulation scheme well approximates the true physical system, uniformly over an infinite time horizon (Thm. 1.15).

The study of large collections of weakly interacting Markov processes and the associated McKean-Vlasov systems has a long history [8,12,20]; also see [2] and references therein. There is an extensive body of work that addresses law of large number behavior (Propagation of Chaos), Central limit theory (normal fluctuations from the mean) and large deviation principles for such models. Most of these results concern the behavior of the system over a finite time horizon. Here we are concerned with uniform in time convergence results (e.g. Thms. 1.9 and 1.15). Such results for interacting diffusions of the form

$$
\mathrm{d} X_{i}(t)=\sqrt{2} d B_{i}(t)-\nabla V\left(X_{i}(t)\right) \mathrm{d} t-\frac{1}{N} \sum_{j=1}^{N} \nabla W\left(X^{i}(t)-X^{j}(t)\right) \mathrm{d} t ; i=1, \ldots, N
$$

have been studied in several works $[5-7,17]$. Here $B^{i}$ are independent Brownian motions and $V, W$ are suitable functions. The key difference from the model studied in the current work (aside from the fact that we work in discrete time) is that, in the above works, the interaction of the $i$ th particle with the system at time $t$ is completely described by the empirical measure $m^{N}(t)=\frac{1}{N} \sum_{i=1}^{N} \delta_{X_{i}(t)}$, whereas in the model studied in our work the interaction at time $t$ involves the whole history $\left\{m^{N}(s)\right\}_{0 \leq s \leq t}$. This can be seen formally from (1.1) on observing that the first equation in the system is driven by the gradient of the interaction function $h$. The precise form of the interaction can be read from equation (1.7). After this work was completed, a recent work [13], where the interaction is given in terms of the empirical measure of the whole history of the particles, was brought to our attention. However, unlike the current work, the above paper is concerned with a finite time asymptotic behavior of the system.

Before we give a formal description of the model, we list some common notation that will be used in this work. For $d \geq 1, \mathbb{R}^{d}$ will denote the $d$-dimensional Euclidean space, the standard norm on which will be denoted by $|\cdot| . \mathbb{N}$ [resp. $\mathbb{N}_{0}$ ] will denote the space of positive [resp. nonnegative] integers. Cardinality of a finite set $G$ will be denoted by $|G|$. For a Polish space $\mathcal{S}, \mathcal{B}(\mathcal{S})$ will denote the Borel sigma field on $\mathcal{S}$ and $\mathcal{P}(\mathcal{S})$ the space of probability measures on $(\mathcal{S}, \mathcal{B}(\mathcal{S}))$. For $x \in \mathcal{S}, \delta_{x}$ will denote the element in $\mathcal{P}(\mathcal{S})$ that puts unit mass at the point $x$. For $\mu \in \mathcal{P}(\mathcal{S})$ and a $\mu$-integrable real measurable map $f$ on $(\mathcal{S}, \mathcal{B}(\mathcal{S}))$, we denote $\int_{\mathcal{S}} f \mathrm{~d} \mu$ as $\mu(f)$ or $\langle\mu, f\rangle$. Similar notations will be used for signed measures. For $\mu \in \mathcal{P}(\mathcal{S}), S_{N}(\mu)$ denotes a random measure defined as $\frac{1}{N} \sum_{k=1}^{N} \delta_{\xi_{k}}$, where $\left\{\xi_{k}\right\}_{k=1}^{N}$ are i.i.d $\mathcal{S}$ valued random variables with distribution $\mu$. We denote the space of real bounded measurable maps on $(\mathcal{S}, \mathcal{B}(\mathcal{S}))$ as $\operatorname{BM}(\mathcal{S})$ and for $f \in \operatorname{BM}(\mathcal{S})$, define $\|f\|_{\infty}=\sup _{x \in \mathcal{S}}|f(x)|$. The space $\left\{f \in \operatorname{BM}(\mathcal{S}):\|f\|_{\infty} \leq 1\right\}$ will be denoted as $\mathcal{B}_{1}(\mathcal{S})$. For a signed measure $\mu$ on $(S, \mathcal{B}(S))$, we define the total variation norm of $\mu$ as $\sup _{f \in \mathcal{B}_{1}(\mathcal{S})}|\mu(f)|$ and denote it by $\|\mu\|_{T V}$. A real function $f$ on $\mathcal{S}$ is said to be $b$-Lipschitz if, for all $x, y \in \mathcal{S},|f(x)-f(y)| \leq b \mathrm{~d}(x, y)$, where $d$ is the metric on $\mathcal{S}$. A transition probability kernel (also referred to as a Markov kernel) on $S$ is a map $\mathcal{T}$ from $S \times \mathcal{B}(S)$ to $[0,1]$ such that for all $x \in S$, $\mathcal{T}(x, \cdot) \in \mathcal{P}(\mathcal{S})$ and for all $A \in \mathcal{B}(\mathcal{S}), \mathcal{T}(\cdot, A) \in \operatorname{BM}(\mathcal{S})$. For $f \in \operatorname{BM}(\mathcal{S})$ and a transition probability kernel $\mathcal{T}$ on $\mathcal{S}$, define $\mathcal{T} f \in \operatorname{BM}(\mathcal{S})$ as $\mathcal{T} f(\cdot)=\int_{\mathcal{S}} f(y) \mathcal{T}(\cdot, \mathrm{d} y)$. For a closed subset $\mathcal{S}_{0}$ of $\mathcal{S}, \mu \in \mathcal{P}\left(\mathcal{S}_{0}\right)$ and a transition kernel $\mathcal{T}$ on $\mathcal{S}$, we define $\mu \mathcal{T} \in \mathcal{P}(\mathcal{S})$ as $\mu \mathcal{T}(A)=\int_{\mathcal{S}_{0}} \mathcal{T}(x, A) \mu(\mathrm{d} x)$. When we are given a reference measure $\ell$ on $\mathcal{S}$, we will denote the density of $\mu \mathcal{T}$ (when it exists) with respect to $\ell$ by $\mathcal{T} \star \mu$.

\subsection{Model for interacting Markovian agents}

The system consists of $N$ particles whose states at time $k \in \mathbb{N}_{0}$ denoted as $X_{1}(k), \ldots, X_{N}(k)$ take value in a compact set $E \subset \mathbb{R}^{d}$ with a non-empty interior. Given that the $j$ th particle is at location $x$ at time instant $k-1$, it moves, independently of other particles, to a set $A \subset \mathcal{B}(E)$ with probability $M^{\eta_{k-1}}(x, A)$. Here $M^{\eta_{k-1}}$ is a transition probability kernel determined by a nonnegative function $\eta_{k-1}$ which represents the "potential field" at time instant $k-1$. Specifically, for any nonnegative function $\Psi: E \rightarrow \mathbb{R}^{+}$, the Markov kernel $M^{\Psi}$ is 
defined as follows.

$$
M^{\Psi}(x, \mathrm{~d} y)=Q(x, \mathrm{~d} y) \mathrm{e}^{-\lambda(\Psi(x)-\Psi(y))_{+}}+Q_{0}(x, \mathrm{~d} y)\left(1-\int_{z \in E} Q(x, \mathrm{~d} z) \mathrm{e}^{-\lambda(\Psi(x)-\Psi(z))_{+}}\right) .
$$

Here $\lambda \in(0, \infty)$ and $Q, Q_{0}$ are two transition probability kernels on $E$. Roughly speaking, to generate a sample from $M^{\Psi}(x, \cdot)$ one follows the following steps:

1. A sample $Y$ is drawn from $Q(x, \cdot)$ and a sample $\tilde{Y}$ is drawn from $Q_{0}(x, \cdot)$.

2. If $\Psi(Y) \geq \Psi(x)$ the sample point $Y$ is accepted. This corresponds to preferential motion in the direction of the gradient of the potential field.

3. If $\Psi(Y)<\Psi(x)$ we accept the sample point $Y$ with probability $\mathrm{e}^{-\lambda(\Psi(x)-\Psi(Y))}$ and take $\tilde{Y}$ with probability $1-\mathrm{e}^{-\lambda(\Psi(x)-\Psi(Y))}$.

The kernel $Q$ captures particle dynamics in absence of the potential field, while $Q_{0}$ can be regarded as the perturbation to the nominal dynamics against the concentration gradient, caused by the potential field. Note that the effect of $Q_{0}$ increases as $\lambda$ becomes larger.

We now describe the evolution of the potential field and its interaction with the particle system. This evolution will aim to capture the essential features of the PDE in (1.1) which are: diffusion, dissipation and dynamic agent input. Let $P$ and $P^{\prime}$ be transition probability kernels on $\mathbb{R}^{d}$ having density with respect to some fixed reference measure $\ell$ on $\mathbb{R}^{d}$ (for example, the Lebesgue measure). Throughout, we will fix a choice of these densities which will ensure that the sequence $\left\{\eta_{k}\right\}$ introduced below is unambiguously defined; also we will always speak of densities as with respect to $\ell$. Given $m \in \mathcal{P}(E)$, define a transition probability kernel $R_{m}$ on $\mathbb{R}^{d}$ as

$$
R_{m}(x, A)=(1-\epsilon) P(x, A)+\epsilon m P^{\prime}(A), \quad x \in \mathbb{R}^{d}, A \in \mathcal{B}\left(\mathbb{R}^{d}\right)
$$

Then given that the potential field at time $k-1$ is described by a nonnegative function $\eta_{k-1}$ on $\mathbb{R}^{d}$ satisfying $\int_{\mathbb{R}^{d}} \eta_{k-1}(x) \mathrm{d} x=1$, and the state values of the $N$ particles are $X_{i}(k-1), i=1, \ldots, N, \eta_{k}$ is defined by the relation.

$$
\eta_{k}(y)=\int_{\mathbb{R}^{d}} \eta_{k-1}(x) R_{m_{k-1}}(x, y) \ell(\mathrm{d} x), m_{k-1}=\frac{1}{N} \sum_{j=1}^{N} \delta_{X_{j}(k-1)} .
$$

In this description, diffusion and advection of the chemical is captured by the kernel $P$, dissipation by the factor $(1-\epsilon)$ and contributions by the agents through the term $\epsilon m_{k-1} P^{\prime}$. We remark that unlike the continuous time setting, here we introduce a single parameter $\epsilon$ rather than two distinct parameters $\alpha$ and $\beta$. This parametrization ensures that the concentration function $\eta_{k}$, for each $k$ is a probability density on $\mathbb{R}^{d}$. The more general setting can be considered as well (although not pursued here) by considering suitably normalized concentration fields.

Thus summarizing, analogous to (1.1), the coupled system of equations describing the evolution of the potential field and particle states is given as follows. Denote by $\mathcal{P}^{*}\left(\mathbb{R}^{d}\right)$ the space of probability measures on $\mathbb{R}^{d}$ that are absolutely continuous with respect to $\ell$. We will identify an element in $\mathcal{P}^{*}\left(\mathbb{R}^{d}\right)$ with its density and denote both by the same symbol. For $N \geq 1$, define $\Pi^{N}: E^{N} \rightarrow \mathcal{P}(E)$ by the relation

$$
\Pi^{N}\left(y_{1}, \ldots, y_{N}\right)=\frac{1}{N} \sum_{i=1}^{N} \delta_{y_{i}}, \quad\left(y_{1}, \ldots, y_{N}\right) \in E^{N} .
$$

Fix random variables $X_{1}, \ldots, X_{N}$ with values in $E$ and $\eta_{0}^{N} \in \mathcal{P}^{*}\left(\mathbb{R}^{d}\right)$. Let $m_{0}^{N}=\Pi^{N}\left(X_{1}, \ldots, X_{N}\right)$. The interacting system of particles and the potential field is described as a family $\left(X^{N}(k), m_{k}^{N}, \eta_{k}^{N}\right)_{k \in N_{0}}$ of $E^{N} \times$ 
$\mathcal{P}(E) \times \mathcal{P}^{*}\left(\mathbb{R}^{d}\right)$ valued random variables on a probability space $(\Omega, \mathcal{F}, \mathbb{P})$, defined recursively as follows. Let $X^{N}(0)=\left(X_{1}, \ldots, X_{N}\right)$ and define $\mathcal{F}_{0}=\sigma\left\{X^{N}(0)\right\}$. For $k \geq 1$

$$
\left\{\begin{array}{l}
\mathbb{P}\left(X^{N}(k) \in A \mid \mathcal{F}_{k-1}\right)=\bigotimes_{j=1}^{N}\left(\delta_{X_{j}^{N}(k-1)} M^{\eta_{k-1}^{N}}\right)(A), A \in \mathcal{B}\left(E^{N}\right) \\
m_{k}^{N}=\Pi^{N}\left(X^{N}(k)\right)=\frac{1}{N} \sum_{j=1}^{N} \delta_{X_{j}^{N}(k)} \\
\eta_{k}^{N}=\eta_{k-1}^{N} R_{m_{k-1}^{N}} \\
\mathcal{F}_{k}=\sigma\left(\eta_{k}^{N}, X^{N}(k)\right) \vee \mathcal{F}_{k-1} .
\end{array}\right.
$$

Along with the $N$ particle system we will also consider the non-linear Markov model which formally corresponds to the $N \rightarrow \infty$ limit of (1.7). Define the map $\Phi: \mathcal{P}(E) \times \mathcal{P}^{*}\left(\mathbb{R}^{d}\right) \rightarrow \mathcal{P}(E) \times \mathcal{P}^{*}\left(\mathbb{R}^{d}\right)$ by the relation

$$
\Phi(m, \eta)=\left(m M^{\eta}, \eta R_{m}\right),(m, \eta) \in \mathcal{P}(E) \times \mathcal{P}^{*}\left(\mathbb{R}^{d}\right) .
$$

Define a sequence of probability measures $\left(m_{n}, \eta_{n}\right)$ on $\mathbb{R}^{d}$ by the following recurrence formula. Fix $\left(m_{0}, \eta_{0}\right) \in$ $\mathcal{P}(E) \times \mathcal{P}^{*}\left(\mathbb{R}^{d}\right)$. For $n \in \mathbb{N}_{0}$

$$
\begin{aligned}
m_{n+1} & =m_{n} M^{\eta_{n}}, \\
\eta_{n+1} & =\eta_{n} R_{m_{n}} .
\end{aligned}
$$

In other words,

$$
\left(m_{n+1}, \eta_{n+1}\right)=\Phi\left(m_{n}, \eta_{n}\right) .
$$

Such a coupled system is similar to what can be found in $[3,4]$.

\subsection{Main results}

We now summarize the main results of this work. For measures $(m, \eta),\left(m^{\prime}, \eta^{\prime}\right) \in \mathcal{P}(E) \times \mathcal{P}^{*}\left(\mathbb{R}^{d}\right)$, we define the norm

$$
\left\|(m, \eta)-\left(m^{\prime}, \eta^{\prime}\right)\right\|=\left\|m-m^{\prime}\right\|_{T V}+\left\|\eta-\eta^{\prime}\right\|_{T V}
$$

We begin with an assumption on the kernels $P, P^{\prime}$.

Assumption 1.1. The kernel $P^{\prime}$ is uniformly bounded on $\mathbb{R}^{d} \times \mathbb{R}^{d}$ and $P^{\prime}(\cdot, y)$ is Lipschitz, uniformly in $y \in \mathbb{R}^{d}$, namely, for some $M_{P^{\prime}}, l_{P^{\prime}} \in(0, \infty)$

$$
\sup _{(x, y) \in \mathbb{R}^{d} \times \mathbb{R}^{d}} P^{\prime}(x, y) \leq M_{P^{\prime}}, \sup _{y \in \mathbb{R}^{d}}\left|P^{\prime}(x, y)-P^{\prime}\left(x^{\prime}, y\right)\right| \leq l_{P^{\prime}}\left|x-x^{\prime}\right|, \forall x, x^{\prime} \in \mathbb{R}^{d} .
$$

The kernel $P$ satisfies, for some $\bar{M}_{P} \in(0, \infty)$,

$$
\sup _{y \in \mathbb{R}^{d}} \int_{x \in \mathbb{R}^{d}} P(x, y) \ell(\mathrm{d} x) \leq \bar{M}_{P} .
$$

The following result establishes the convergence of the stochastic system to the non-linear deterministic dynamical system over any fixed time horizon.

Theorem 1.2. Suppose that Assumption 1.1 holds. Also suppose that

$$
\left\|\eta_{0}^{N}-\eta_{0}\right\|_{\infty} \rightarrow 0 \text { and } \sup _{f \in \mathcal{B}_{1}(E)} \mathbb{E}\left(\left|\left\langle m_{0}-m_{0}^{N}, f\right\rangle\right|\right) \rightarrow 0 \text { as } N \rightarrow \infty .
$$

Then, $\forall k \in \mathbb{N}_{0}$,

$$
\sup _{f \in \mathcal{B}_{1}(E)} \mathbb{E}\left(\left|\left\langle m_{k}^{N}-m_{k}, f\right\rangle\right|+\left\|\eta_{k}^{N}-\eta_{k}\right\|_{\infty}\right) \underset{N \rightarrow+\infty}{\longrightarrow} 0
$$


We remark that $\mathbb{E}\left\|\eta_{k}^{N}-\eta_{k}\right\|_{\infty} \rightarrow 0$ implies, by an application of Scheffe's theorem, that $\mathbb{E}\left\|\eta_{k}^{N}-\eta_{k}\right\|_{T V} \rightarrow 0$. This observation will be used in the Proof of Theorem 1.9.

As an immediate consequence of this result we obtain the following propagation of chaos result. For $p \geq 1$, denote by $m_{k}^{N, p}$ the probability law of $\left(X_{1}^{N}(k), \ldots, X_{p}^{N}(k)\right)$ on $E^{p}$.

Corollary 1.3. Under Assumption 1.1, for every $p \in \mathbb{N}, m_{k}^{N, p}$ converges weakly to $m_{k}^{\otimes p}$, as $N \rightarrow \infty$.

We now study time asymptotic properties of the system. We begin with two basic assumptions. The first is on the kernel $Q$.

Assumption 1.4. There exist $\epsilon_{Q} \in(0,1)$ and $\ell_{1} \in \mathcal{P}(E)$ such that $\forall x \in E, \forall A \in \mathcal{B}(E), Q(x, A) \geq \epsilon_{Q} \ell_{1}(A)$.

It is well known that under Assumption 1.4, $Q$ is $\left(1-\epsilon_{Q}\right)$-contracting for the total variation norm (see Lem. A.1 in Appendix for a proof), i.e.

$$
\left\|\mu Q-\mu^{\prime} Q\right\|_{T V} \leq\left(1-\epsilon_{Q}\right)\left\|\mu-\mu^{\prime}\right\|_{T V}, \forall \mu, \mu^{\prime} \in \mathcal{P}(E) .
$$

Next, we will make the following assumption on the kernels $P, P^{\prime}$.

\section{Assumption 1.5.}

1. There exists $\beta\left(P^{\prime}\right) \in(0,1)$ such that for all $m, m^{\prime} \in \mathcal{P}(E)$,

$$
\left\|m P^{\prime}-m^{\prime} P^{\prime}\right\|_{T V} \leq \beta\left(P^{\prime}\right)\left\|m-m^{\prime}\right\|_{T V} .
$$

2. The kernel $P$ is uniformly bounded on $\mathbb{R}^{d} \times \mathbb{R}^{d}$, i.e. for some $M_{P} \in(0, \infty)$

$$
\sup _{(x, y) \in \mathbb{R}^{d} \times \mathbb{R}^{d}} P(x, y) \leq M_{P}
$$

We begin with the following result on the fixed points of the dynamical system (1.10).

Theorem 1.6. Suppose that Assumptions 1.1-1.5 hold. Then there are $\lambda_{0}, \epsilon_{0} \in(0, \infty)$ such that for all $\lambda \leq \lambda_{0}$ and $\epsilon \leq \epsilon_{0}$, $\Phi$ has a unique fixed point in $\mathcal{P}(E) \times \mathcal{P}^{*}\left(\mathbb{R}^{d}\right)$.

Recall the sequence $\left(m_{n}, \eta_{n}\right)$ defined through equation (1.10) recursively with a fixed initial pair $\left(m_{0}, \eta_{0}\right) \in$ $\mathcal{P}(E) \times \mathcal{P}^{*}\left(\mathbb{R}^{d}\right)$. Also recall the collection of random variables $\left(X^{N}(k), m_{k}^{N}, \eta_{k}^{N}\right)_{k \in \mathbb{N}_{0}}$ with values in $E^{N} \times$ $\mathcal{P}(E) \times \mathcal{P}^{*}\left(\mathbb{R}^{d}\right)$ defined in (1.7) starting with a fixed $\eta_{0}^{N} \in \mathcal{P}^{*}\left(\mathbb{R}^{d}\right)$ and $X_{1}, \ldots, X_{N} \in E$.

We now consider uniform in time convergence of the stochastic system to the non-linear dynamical system. For that we will make the following additional assumptions.

\section{Assumption 1.7.}

1. $P(\cdot, y)$ is Lipschitz uniformly in $y \in \mathbb{R}^{d}$ namely, for some $l_{P} \in(0, \infty)$

$$
\sup _{y \in \mathbb{R}^{d}}\left|P(x, y)-P\left(x^{\prime}, y\right)\right| \leq l_{P}\left|x-x^{\prime}\right|, \forall x, x^{\prime} \in \mathbb{R}^{d} .
$$

2. There exist $p \in \mathcal{P}\left(\mathbb{R}^{d}\right)$ and $\tilde{l}_{P^{\prime}}$ such that $\forall x, x^{\prime} \in E, A \in \mathcal{B}\left(\mathbb{R}^{d}\right)$,

$$
\left|P^{\prime}(x, A)-P^{\prime}\left(x^{\prime}, A\right)\right| \leq \tilde{l}_{P^{\prime}}\left|x-x^{\prime}\right| p(A) .
$$

3. $P(x, \cdot), P^{\prime}(x, \cdot)$ are Lipschitz uniformly in $x \in \mathbb{R}^{d}$ namely, for some $\bar{l}_{P . P^{\prime}} \in(0, \infty)$

$$
\sup _{x \in \mathbb{R}^{d}} \max \left\{\left|P(x, y)-P\left(x, y^{\prime}\right)\right|,\left|P^{\prime}(x, y)-P^{\prime}\left(x, y^{\prime}\right)\right|\right\} \leq \bar{l}_{P, P^{\prime}}\left|y-y^{\prime}\right|, \forall y, y^{\prime} \in \mathbb{R}^{d} .
$$


4. For some $\bar{M}_{P, P^{\prime}} \in(0, \infty)$

$$
\sup _{y \in \mathbb{R}^{d}} \max \left\{\int_{x \in \mathbb{R}^{d}} P(x, y) l(\mathrm{~d} x), \int_{x \in \mathbb{R}^{d}} P^{\prime}(x, y) l(\mathrm{~d} x)\right\} \leq \bar{M}_{P, P^{\prime}} .
$$

Denote by $\ell_{E}$ the restriction of the measure $l$ to $E$.

Assumption 1.8. For all $x \in E, Q(x,$.$) and Q_{0}(x,$.$) have densities with respect to \ell_{E}$. The densities are bounded on $E \times E$, namely for some $M_{Q, Q_{0}} \in(0, \infty)$

$$
\sup _{(x, y) \in E \times E} \max \left\{Q(x, y), Q_{0}(x, y)\right\} \leq M_{Q, Q_{0}} .
$$

Furthermore, for some $l_{Q, Q_{0}} \in(0, \infty)$ and $\bar{p} \in \mathcal{P}(E)$

$$
\max \left\{\left|Q(x, A)-Q\left(x^{\prime}, A\right)\right|,\left|Q_{0}(x, A)-Q_{0}\left(x^{\prime}, A\right)\right|\right\} \leq l_{Q, Q_{0}}\left|x-x^{\prime}\right| \bar{p}(A) \quad \forall x, x^{\prime} \in \quad E, A \in \mathcal{B}(E) .
$$

Theorem 1.9. Suppose that Assumptions 1.1-1.8 hold. Let $\epsilon_{0}, \lambda_{0}$ be as in Theorem 1.6. Then, whenever $\epsilon \leq \epsilon_{0}$ and $\lambda \leq \lambda_{0}$, we have:

(i) For some $c_{0} \in(0, \infty)$,

$$
\forall \delta>0, \exists N_{0}, n_{0} \in \mathbb{N} \text { such that } \forall n \geq n_{0} \text { and } N \geq N_{0}, \sup _{\|f\|_{\infty} \leq 1} \mathbb{E}\left(\left|\left\langle m_{n}^{N}-m_{n}, f\right\rangle\right|+\left\|\eta_{n}^{N}-\eta_{n}\right\|_{T V}\right) \leq c_{0} \delta .
$$

(ii) If $\left\|\eta_{0}^{N}-\eta_{0}\right\|_{\infty} \rightarrow 0$ and $\sup _{f \in \mathcal{B}_{1}(E)} \mathbb{E}\left(\left|\left\langle m_{0}-m_{0}^{N}, f\right\rangle\right|\right) \rightarrow 0$ as $N \rightarrow \infty$, then for some $c_{1} \in(0, \infty)$

$$
\forall \delta>0, \exists N_{0} \in \mathbb{N} \text { such that } \forall n \text { and } N \geq N_{0}, \sup _{\|f\|_{\infty} \leq 1} \mathbb{E}\left(\left|\left\langle m_{n}^{N}-m_{n}, f\right\rangle\right|+\left\|\eta_{n}^{N}-\eta_{n}\right\|_{T V}\right) \leq c_{1} \delta .
$$

As an immediate consequence of Theorems 1.6 and 1.9 we have that under suitable conditions $\left(m_{k}^{N}, \eta_{k}^{N}\right)$ approaches the unique fixed point of $\Phi$ as $k \rightarrow \infty$ and $N \rightarrow \infty$. Namely,

Corollary 1.10. Suppose that Assumptions 1.1-1.8 hold. Let $\epsilon_{0}, \lambda_{0}$ be as in Theorem 1.6. Fix $\epsilon \in\left(0, \epsilon_{0}\right)$ and $\lambda \in\left(0, \lambda_{0}\right)$. Denote the corresponding unique fixed point of $\Phi$ by $\left(m_{\infty}, \eta_{\infty}\right)$. Then,

$$
\begin{aligned}
& \limsup _{n \rightarrow \infty} \limsup _{N \rightarrow \infty} \sup _{\|f\|_{\infty} \leq 1} \mathbb{E}\left(\left|\left\langle m_{n}^{N}-m_{\infty}, f\right\rangle\right|+\left\|\eta_{n}^{N}-\eta_{\infty}\right\|_{T V}\right) \\
= & \limsup _{N \rightarrow \infty} \limsup _{n \rightarrow \infty} \sup _{\|f\|_{\infty} \leq 1} \mathbb{E}\left(\left|\left\langle m_{n}^{N}-m_{\infty}, f\right\rangle\right|+\left\|\eta_{n}^{N}-\eta_{\infty}\right\|_{T V}\right) \\
= & 0 .
\end{aligned}
$$

Simulation of the stochastic system in (1.7) or numerical computation of the paths of the deterministic dynamical system (1.10) can in general be quite hard and so it is of interest to develop good approximation schemes. One flexible and appealing approach is to approximate the measures, $\eta_{n}^{N}$ in the first case and the measures $\left(\eta_{n}, m_{n}\right)$ in the second case, by discrete probability distributions through a collection of evolving particles. We will consider one such particle scheme in this work and show that, under conditions, the error between the dynamical system in (1.10) and the one obtained through a particle approximation can be controlled uniformly in time. We will also prove a similar result for the error between the actual physical stochastic system and the one obtained through particle approximations of $\eta_{n}^{N}$. For these results we will make the following additional assumption. 
Assumption 1.11. $P, P^{\prime}$ are Gaussian kernels and $\ell$ is the Lebesgue measure on $\mathbb{R}^{d}$.

Note that if $P, P^{\prime}$ satisfy Assumption 1.11, then they also satisfy Assumptions 1.1, 1.5 and 1.7 (for Assump. 1.5 see Lem. A.6).

We propose the following particle system for the system (1.10) that starts from $\left(m_{0}, \eta_{0}\right) \in \mathcal{P}(E) \times \mathcal{P}^{*}\left(\mathbb{R}^{d}\right)$. We assume that we can draw samples from $\left(m_{0}, \eta_{0}\right)$. We remark that although for Gaussian kernels, the integral in (1.5) can be computed analytically as a mixture of Gaussian densities, the computation becomes numerically unfeasible since the number of terms in this mixture grows linearly over time.

Denote by $\left(\tilde{X}_{1}^{N}(0), \ldots, \tilde{X}_{N}^{N}(0)\right)$ a sample of size $N$ from $m_{0}$. Let $\tilde{m}_{0}^{N}=\Pi^{N}\left(\tilde{X}_{1}^{N}(0), \ldots, \tilde{X}_{N}^{N}(0)\right)$. The approximating particle system is given as a family $\left(\tilde{X}^{N}(k), m_{k}^{N}, \eta_{k}^{N}\right)_{k \in \mathbb{N}_{0}}$ of $E^{N} \times \mathcal{P}(E) \times \mathcal{P}^{*}\left(\mathbb{R}^{d}\right)$ valued random variables on some probability space $(\Omega, \mathcal{F}, \mathbb{P})$, defined recursively as follows. Set $\tilde{X}^{N}(0)=\left(\tilde{X}_{1}^{N}(0), \ldots, \tilde{X}_{N}^{N}(0)\right)$, $\tilde{\eta}_{0}^{N}=\eta_{0}, \mathcal{F}_{0}=\sigma\left(\tilde{X}^{N}(0)\right)$. For $k \geq 1$

$$
\left\{\begin{array}{l}
\mathbb{P}\left(\tilde{X}^{N}(k) \in A \mid \mathcal{F}_{k-1}\right)=\bigotimes_{j=1}^{N}\left(\delta_{\tilde{X}_{j}^{N}(k-1)} M^{\tilde{\eta}_{k-1}^{N}}\right)(A), A \in \mathcal{B}\left(E^{N}\right) \\
\tilde{m}_{k}^{N}=\frac{1}{N} \sum_{j=1}^{N} \delta_{\tilde{X}_{j}^{N}(k)} \\
\tilde{\eta}_{k}^{N}=(1-\epsilon)\left(S^{N}\left(\tilde{\eta}_{k-1}^{N}\right) P\right)+\epsilon\left(\tilde{m}_{k-1}^{N} P^{\prime}\right) \\
\mathcal{F}_{k}=\sigma\left(\tilde{X}^{N}(k), \tilde{\eta}_{k}^{N}\right) \vee \mathcal{F}_{k-1} .
\end{array}\right.
$$

Here $S^{N}\left(\tilde{\eta}_{k-1}^{N}\right)$ is the random probability measure defined as $\frac{1}{N} \sum_{i=1}^{N} \delta_{Y_{i}^{N}(k)}$ where $Y_{1}^{N}(k), \cdots Y_{N}^{N}(k)$, conditionally on $\mathcal{F}_{k-1}$, are i.i.d. distributed according to $\tilde{\eta}_{k-1}^{N}$.

Notice that under the above Gaussian assumption, $\tilde{\eta}_{k}^{N}$ is a mixture of $2 N$ - Gaussian random variables for any $k \geq 1$, so we can compute numerically its density at any point. So (1.12) defines an implementable particle scheme. We first consider convergence over a fixed time horizon.

Theorem 1.12. Under Assumption 1.11, we have for all $k$,

$$
\sup _{f \in \mathcal{B}_{1}(E)} \mathbb{E}\left(\left|\left\langle\tilde{m}_{k}^{N}-m_{k}, f\right\rangle\right|+\left\|\tilde{\eta}_{k}^{N}-\eta_{k}\right\|_{T V}+\left\|\tilde{\eta}_{k}^{N}-\eta_{k}\right\|_{\infty}\right) \underset{N \rightarrow+\infty}{\longrightarrow} 0 .
$$

We now consider uniform in time convergence of the approximation scheme.

Theorem 1.13. Suppose that Assumptions 1.4-1.11 hold. Then there exists $c_{1} \in(0, \infty)$ such that for $\epsilon \leq \epsilon_{0}$, $\lambda \leq \lambda_{0}$ and $\left(\eta_{0}, m_{0}\right) \in \mathcal{P}^{*}\left(\mathbb{R}^{d}\right) \times \mathcal{P}(E)$, the sequences defined in (1.12) are such that

$$
\forall \delta>0, \exists N_{0} \text { such that } \forall n \text { and } N \geq N_{0}, \sup _{f \in \mathcal{B}_{1}(E)} \mathbb{E}\left(\left|\left\langle\tilde{m}_{n}^{N}-m_{n}, f\right\rangle\right|+\left\|\tilde{\eta}_{n}^{N}-\eta_{n}\right\|_{T V}\right)<c_{1} \delta .
$$

As corollaries of Theorems 1.2-1.13 we have the following results.

Theorem 1.14. Under Assumption 1.4 and assumptions of Theorem 1.2, we have for all $k \geq 0$,

$$
\sup _{f \in \mathcal{B}_{1}(E)} \mathbb{E}\left(\left|\left\langle m_{k}^{N}-\tilde{m}_{k}^{N}, f\right\rangle\right|+\left\|\eta_{k}^{N}-\tilde{\eta}_{k}^{N}\right\|_{T V}\right) \underset{N \rightarrow+\infty}{\longrightarrow} 0 .
$$

Theorem 1.15. Under assumptions $1.4,1.8$ and 1.11 there exists $c_{1} \in(0, \infty)$ such that for $\epsilon \leq \epsilon_{0}, \lambda \leq \lambda_{0}$,

$$
\forall \delta>0, \exists N_{0}, n_{0} \in \mathbb{N} \text { such that } \forall n \geq n_{0} \text { and } N \geq N_{0}, \sup _{\|f\|_{\infty} \leq 1} \mathbb{E}\left(\left|\left\langle m_{n}-\tilde{m}_{n}^{N}, f\right\rangle\right|+\left\|\eta_{n}^{N}-\tilde{\eta}_{n}^{N}\right\|_{T V}\right)<c_{1} \delta .
$$

We remark that in approximating $\eta_{k}$ by $\tilde{\eta}_{k}^{N}$ and $m_{k}$ by $\tilde{m}_{k}^{N}$ we have taken for simplicity $N$ particles for both approximations. Although not pursued here, one can similarly analyse particle schemes where the number of particles for approximating $\eta_{k}$ is different from that used for approximating $m_{k}$. 


\section{ProOFS}

\subsection{Convergence over a finite time horizon}

In this subsection we prove Theorem 1.2 and Corollary 1.3. We begin with a lemma that will be used several times in this work. Proof is immediate from Ascoli-Arzela theorem.

Lemma 2.1. Let $K$ be a compact subset of $\mathbb{R}^{d}$ and let for $a, b \in(0, \infty), F_{a, b}(K)$ be the collection of all functions $f: K \rightarrow \mathbb{R}$ such that $\|f\|_{\infty} \leq a$ and $f$ is $b$-Lipschitz. Then for every $\delta>0$ there exists a finite subset $F_{a, b}^{\delta}(K)$ of $F_{a, b}(K)$ such that for every signed measure $\mu$ on $K$

$$
\left.\sup _{f \in F_{a, b}(K)}|\langle\mu, f\rangle|\right) \leq \max _{g \in F_{a, b}^{\delta}(K)}|\langle\mu, g\rangle|+\delta\|\mu\|_{T V} .
$$

Frequently, when clear from context, we will suppress $K$ in the notation when writing $F, F^{\delta}$. The following elementary estimate will be used several times.

Lemma 2.2. For all $x, y, x^{\prime}, y^{\prime} \in \mathbb{R}$,

$$
\left|\mathrm{e}^{-\lambda(x-y)_{+}}-\mathrm{e}^{-\lambda\left(x^{\prime}-y^{\prime}\right)+}\right| \leq \lambda\left|x-x^{\prime}\right|+\lambda\left|y-y^{\prime}\right| .
$$

Proof of Theorem 1.2. We proceed recursively. Note that, by assumption, (1.11) holds for $k=0$. Suppose now that (1.11) holds for some fixed $k \in \mathbb{N}_{0}$. Then

$$
m_{k+1}^{N}-m_{k+1}=m_{k+1}^{N}-m_{k}^{N} M^{\eta_{k}^{N}}+m_{k}^{N} M^{\eta_{k}^{N}}-m_{k}^{N} M^{\eta_{k}}+m_{k}^{N} M^{\eta_{k}}-m_{k} M^{\eta_{k}} .
$$

From Lemma A.5 in the Appendix

$$
\sup _{f \in \mathcal{B}_{1}(E)} \mathbb{E}\left(\left|\left\langle m_{k+1}^{N}-m_{k}^{N} M^{\eta_{k}^{N}}, f\right\rangle\right|\right) \leq \frac{2}{\sqrt{N}} \underset{N \rightarrow+\infty}{\longrightarrow} 0 .
$$

Note that for all $f \in \mathcal{B}_{1}(E), m \in \mathcal{P}(E)$ and $\eta, \eta^{\prime} \in \mathcal{P}^{*}\left(\mathbb{R}^{d}\right)$

$$
\begin{aligned}
\left|m M^{\eta}(f)-m M^{\eta^{\prime}}(f)\right|= & \mid \int_{x, y \in E} m(\mathrm{~d} x) Q(x, \mathrm{~d} y)\left(\mathrm{e}^{-\lambda(\eta(x)-\eta(y))_{+}}-\mathrm{e}^{-\lambda\left(\eta^{\prime}(x)-\eta^{\prime}(y)\right)_{+}}\right) f(y)+\int m(\mathrm{~d} x) Q_{0}(x, \mathrm{~d} y) \\
& \times\left(\int_{z \in E} Q(x, \mathrm{~d} z)\left(-\mathrm{e}^{-\lambda(\eta(x)-\eta(z))_{+}}+\mathrm{e}^{\lambda\left(\eta^{\prime}(x)-\eta^{\prime}(z)\right)_{+}}\right) f(y) \mid .\right.
\end{aligned}
$$

Thus by Lemma 2.2 and since (1.11) holds for $k$, we have, $\forall f \in \mathcal{B}_{1}(E)$,

$$
\mathbb{E}\left(\left|\left\langle m_{k}^{N} M^{\eta_{k}^{N}}-m_{k}^{N} M^{\eta_{k}}, f\right\rangle\right|\right) \leq 4 \lambda \mathbb{E}\left(\left\|\eta_{k}^{N}-\eta_{k}\right\|_{\infty}\right) \underset{N \rightarrow+\infty}{\longrightarrow} 0 .
$$

Also, for $f \in \mathcal{B}_{1}(E)$,

$$
\left\langle m_{k}^{N} M^{\eta_{k}}-m_{k} M^{\eta_{k}}, f\right\rangle=\left\langle m_{k}^{N}-m_{k}, M^{\eta_{k}} f\right\rangle .
$$

Since $f \in \mathcal{B}_{1}(E)$, we have that $M^{\eta_{k}} f \in \mathcal{B}_{1}(E)$ and so

$$
\mathbb{E}\left(\left|\left\langle m_{k} M^{\eta_{k}}-m_{k}^{N} M^{\eta_{k}}, f\right\rangle\right|\right) \leq \sup _{g \in \mathcal{B}_{1}(E)} \mathbb{E}\left(\mid\left\langle m_{k}-m_{k}^{N}, g\right\rangle\right) .
$$

Once again using the fact that (1.11) holds for $k$, we have from the above inequality, that

$$
\sup _{f \in \mathcal{B}_{1}(E)} \mathbb{E}\left(\left|\left\langle m_{k} M^{\eta_{k}}-m_{k}^{N} M^{\eta_{k}}, f\right\rangle\right|\right) \underset{N \rightarrow+\infty}{\longrightarrow} 0,
$$


and combining the above convergence statements

$$
\sup _{f \in \mathcal{B}_{1}(E)} \mathbb{E}\left(\mid\left\langle m_{k+1}^{N}-m_{k+1}, f>\right|\right) \underset{N \rightarrow+\infty}{\longrightarrow} 0 .
$$

Next

$$
\eta_{k+1}^{N}(x)-\eta_{k+1}(x)=(1-\epsilon)\left(P \star\left(\eta_{k}^{N}-\eta_{k}\right)\right)(x)+\epsilon\left(P^{\prime} \star\left(m_{k}^{N}-m_{k}\right)\right)(x), x \in \mathbb{R}^{d},
$$

where, recall that for $\mu \in \mathcal{P}\left(\mathbb{R}^{d}\right), P \star \mu$ is a function on $\mathbb{R}^{d}$ defined as $P \star \mu(x)=\int_{\mathbb{R}^{d}} P(y, x) \mu(\mathrm{d} y), x \in \mathbb{R}^{d}$. $P^{\prime} \star \mu$ for $\mu \in \mathcal{P}(E)$ is defined similarly. Using Assumption 1.1 we have

$$
\mathbb{E}\left(\left\|P \star\left(\eta_{k}^{N}-\eta_{k}\right)\right\|_{\infty}\right) \leq \bar{M}_{P} \mathbb{E}\left(\left\|\eta_{k}^{N}-\eta_{k}\right\|_{\infty}\right) \underset{N \rightarrow+\infty}{\longrightarrow} 0 .
$$

Finally, for an arbitrary $\delta>0$, we have from Lemma 2.1 and Assumption 1.1 that

$$
\left\|P^{\prime} \star\left(m_{k}^{N}-m_{k}\right)\right\|_{\infty}=\sup _{y \in \mathbb{R}^{d}}\left|\int_{x \in E}\left(m_{k}^{N}(\mathrm{~d} x)-m_{k}(\mathrm{~d} x)\right) P^{\prime}(x, y)\right| \leq\left(\max _{g \in F_{M_{P^{\prime}, l} P^{\prime}}^{\prime}}\left|\left\langle m_{k}^{N}-m_{k}, g\right\rangle\right|+2 \delta\right),
$$

where $F^{\delta}$ is the finite family as in Lemma 2.1 associated with $K=E$. Recalling that (1.11) holds for $k$ and noting that $\delta>0$ is arbitrary and the family $F^{\delta}\left(M_{P^{\prime}}, l_{P^{\prime}}\right)$ is finite, we have from the above two estimates that

$$
\mathbb{E}\left(\left\|\eta_{k+1}^{N}-\eta_{k}\right\|_{\infty}\right) \underset{N \rightarrow+\infty}{\longrightarrow} 0 .
$$

The result follows.

Proof of Corollary 1.3. We can apply Proposition 2.2 (i) page 177 of [20] to get that $\forall k, p \in \mathbb{N}, \forall \phi_{1}, \ldots, \phi_{p} \in$ $C_{b}(E), \mathbb{E}\left(m_{k}^{N, p}\left(\phi_{1} \otimes \cdots \otimes \phi_{p}\right)\right) \underset{N \rightarrow+\infty}{\longrightarrow} m_{k}^{\otimes p}\left(\phi_{1} \otimes \cdots \otimes \phi_{p}\right)$. We conclude by a denseness argument.

\subsection{Existence and uniqueness of fixed points}

In this section we will prove Theorem 1.6. Some of the techniques in this section are comparable to what can be found in [4].

For $g: \mathbb{R}^{d} \rightarrow \mathbb{R}$, let osc $(g)=\sup _{x, y \in \mathbb{R}^{d}}|g(x)-g(y)|$. For $n>1$, define $\Phi^{n}$ recursively as $\Phi^{n}=\Phi \circ \Phi^{n-1}$, where $\Phi^{1}=\Phi$. Let $M_{P, P^{\prime}}=\max \left\{M_{P}, M_{P^{\prime}}\right\}$. We begin with the following lemma.

Lemma 2.3. Suppose that Assumptions 1.1-1.5 hold. There are $\epsilon_{0}, \lambda_{0} \in(0,1)$ such that for any $\epsilon \leq \epsilon_{0}$ and $\lambda \leq \lambda_{0}$ there exists $\theta \in(0,1)$ such that $\forall n$

$$
\|\left(\Phi^{n}\left(m_{0}, \eta_{0}\right)-\Phi^{n}\left(m_{0}^{\prime}, \eta_{0}^{\prime}\right) \| \leq 4 \theta^{n-1},\right.
$$

for all $\left(m_{0}, \eta_{0}\right),\left(m_{0}^{\prime}, \eta_{0}^{\prime}\right) \in \mathcal{P}(E) \times \mathcal{P}^{*}\left(\mathbb{R}^{d}\right)$.

Proof. Note that from Assumption $1.5(1)$, for any $(m, \eta) \in \mathcal{P}(E) \times \mathcal{P}^{*}\left(\mathbb{R}^{d}\right)$

$$
\left\|\eta R_{m}-\eta^{\prime} R_{m^{\prime}}\right\| T V \leq(1-\epsilon)\left\|\eta-\eta^{\prime}\right\| T V+\epsilon \beta\left(P^{\prime}\right)\left\|m-m^{\prime}\right\| T V .
$$

and by Lemma A.2 in the Appendix,

$$
\left\|m M^{\eta}-m^{\prime} M^{\eta}\right\|_{T V} \leq\left(1-\epsilon_{Q} \mathrm{e}^{-\lambda \operatorname{osc}(\eta)}\right)\left\|m-m^{\prime}\right\|_{T V} .
$$

Fix $\left(m_{0}, \eta_{0}\right),\left(m_{0}^{\prime}, \eta_{0}^{\prime}\right) \in \mathcal{P}(E) \times \mathcal{P}^{*}\left(\mathbb{R}^{d}\right)$ and let $\left(m_{n}, \eta_{n}\right)=\Phi^{n}\left(m_{0}, \eta_{0}\right),\left(m_{n}^{\prime}, \eta_{n}^{\prime}\right)=\Phi^{n}\left(m_{0}^{\prime}, \eta_{0}^{\prime}\right)$. From Assumptions 1.1 and 1.5 , we have $\forall n \geq 1$,

$$
\operatorname{osc}\left(\eta_{n}\right) \leq M_{P, P^{\prime}}, \operatorname{osc}\left(\eta_{n}^{\prime}\right) \leq M_{P, P^{\prime}}
$$


and therefore for $k \geq 2$,

$$
\left\|m_{k-1} M^{\eta_{k-1}^{\prime}}-m_{k-1}^{\prime} M^{\eta_{k-1}^{\prime}}\right\|_{T V} \leq\left(1-\epsilon_{Q} \mathrm{e}^{-\lambda M_{P, P^{\prime}}}\right)\left\|m_{k-1}-m_{k-1}^{\prime}\right\|_{T V} .
$$

For $k \geq 2$ the measures $m_{k-2} P^{\prime}$ and $m_{k-2}^{\prime} P^{\prime}$ have densities and these densities satisfy $\forall y$

$$
\left|m_{k-2} P^{\prime}(y)-m_{k-2}^{\prime} P^{\prime}(y)\right|=\left|\int_{x \in E} m_{k-2}(\mathrm{~d} x) P^{\prime}(x, y)-m_{k-2}^{\prime}(\mathrm{d} x) P^{\prime}(x, y)\right| \leq M_{P, P^{\prime}}\left\|m_{k-2}-m_{k-2}^{\prime}\right\|_{T V} .
$$

The measures $\eta_{k-2} P$ and $\eta_{k-2}^{\prime} P$ have densities as well and these densities satisfy $\forall y$,

$$
\left|\eta_{k-2} P(y)-\eta_{k-2}^{\prime} P(y)\right|=\left|\int_{x \in E}\left(\eta_{k-2}(\mathrm{~d} x) P(x, y)-\eta_{k-2}^{\prime}(\mathrm{d} x) P(x, y)\right)\right| \leq M_{P, P^{\prime}}\left\|\eta_{k-2}-\eta_{k-2}^{\prime}\right\|_{T V} .
$$

So, $\forall x, y \in \mathbb{R}^{d}$, using (1.9), (2.6), (2.7),

$$
\begin{aligned}
\left|\mathrm{e}^{-\lambda\left(\eta_{k-1}(x)-\eta_{k-1}(y)\right)_{+}}-\mathrm{e}^{-\lambda\left(\eta_{k-1}^{\prime}(x)-\eta_{k-1}^{\prime}(y)\right)_{+}}\right| & \leq \lambda\left|\eta_{k-1}(x)-\eta_{k-1}^{\prime}(x)\right|+\lambda\left|\eta_{k-1}(y)-\eta_{k-1}^{\prime}(y)\right| \\
& \leq 2 \lambda M_{P, P^{\prime}}\left((1-\epsilon)\left\|\eta_{k-2}-\eta_{k-2}^{\prime}\right\|_{T V}+\epsilon\left\|m_{k-2}-m_{k-2}^{\prime}\right\|_{T V}\right) .
\end{aligned}
$$

By (2.1) we then have, for $f \in \mathcal{B}_{1}(E)$,

$$
\left|m_{k-1} M^{\eta_{k-1}}(f)-m_{k-1} M^{\eta_{k-1}^{\prime}}(f)\right| \leq 4 \lambda M_{P, P^{\prime}}\left((1-\epsilon)\left\|\eta_{k-2}-\eta_{k-2}^{\prime}\right\|_{T V}+\epsilon\left\|m_{k-2}-m_{k-2}^{\prime}\right\|_{T V}\right) .
$$

By (2.5), (2.9), we get

$$
\begin{aligned}
\left\|m_{k}-m_{k}^{\prime}\right\|_{T V} & \leq\left\|m_{k-1} M^{\eta_{k-1}}-m_{k-1} M^{\eta_{k-1}^{\prime}}\right\|_{T V}+\left\|m_{k-1} M^{\eta_{k-1}^{\prime}}-m_{k-1}^{\prime} M^{\eta_{k-1}^{\prime}}\right\|_{T V} \\
& \leq 4 \lambda M_{P, P^{\prime}}\left((1-\epsilon)\left\|\eta_{k-2}-\eta_{k-2}^{\prime}\right\|_{T V}+\epsilon\left\|m_{k-2}-m_{k-2}^{\prime}\right\|_{T V}\right) \\
& +\left(1-\epsilon_{Q} \mathrm{e}^{\left.-\lambda M_{P, P^{\prime}}\right)\left\|m_{k-1}-m_{k-1}^{\prime}\right\|_{T V}}\right.
\end{aligned}
$$

and combining this with $(2.2)$, we have

$$
\begin{aligned}
\left\|\left(m_{k}, \eta_{k}\right)-\left(m_{k}^{\prime}, \eta_{k}^{\prime}\right)\right\| & \leq 4 \lambda M_{P, P^{\prime}}\left((1-\epsilon)\left\|\eta_{k-2}-\eta_{k-2}^{\prime}\right\|_{T V}+\epsilon\left\|m_{k-2}-m_{k-2}^{\prime}\right\|_{T V}\right) \\
& +\left(1-\epsilon_{Q} \mathrm{e}^{\left.-\lambda M_{P, P^{\prime}}\right)\left\|m_{k-1}-m_{k-1}^{\prime}\right\|_{T V}}\right. \\
& +(1-\epsilon)\left\|\eta_{k-1}-\eta_{k-1}^{\prime}\right\|_{T V}+\epsilon \beta\left(P^{\prime}\right)\left\|m_{k-1}-m_{k-1}^{\prime}\right\|_{T V} .
\end{aligned}
$$

We can find $\epsilon_{0} \in(0,1), \lambda_{0} \in(0, \infty)$ such that for all $\epsilon \in\left(0, \epsilon_{0}\right), \lambda \in\left(0, \lambda_{0}\right)$, there exists a $\theta \equiv \theta(\epsilon, \lambda) \in(0,1)$, such that

$$
\frac{\sup \left((1-\epsilon),\left(1-\epsilon_{Q} \mathrm{e}^{\left.\left.-\lambda M_{P, P^{\prime}}\right)+\epsilon \beta\left(P^{\prime}\right)\right)}\right.\right.}{\theta}+\frac{4 \lambda M_{P, P^{\prime}}}{\theta^{2}} \leq 1
$$

Note that

$$
\begin{aligned}
& \left\|\left(m_{0}, \eta_{0}\right)-\left(m_{0}^{\prime}, \eta_{0}^{\prime}\right)\right\| \leq 4 \theta^{-1}, \\
& \left\|\left(m_{1}, \eta_{1}\right)-\left(m_{1}^{\prime}, \eta_{1}^{\prime}\right)\right\| \leq 4 .
\end{aligned}
$$

We then have by recurrence that for $\epsilon \in\left(0, \epsilon_{0}\right), \lambda \in\left(0, \lambda_{0}\right),\left\|\left(m_{n}, \eta_{n}\right)-\left(m_{n}^{\prime}, \eta_{n}^{\prime}\right)\right\| \leq 4 \theta^{n-1}, \forall n \in \mathbb{N}_{0}$.

With $\epsilon_{0}, \lambda_{0}$ and $\theta(\epsilon, \lambda) \equiv \theta$ as in the above lemma, let $\kappa=\frac{4 \lambda M_{P, P^{\prime}}}{\theta}$. Then from the estimate in (2.11) it follows that, for all $k \geq 2$ and $\epsilon \in\left(0, \epsilon_{0}\right), \lambda \in\left(0, \lambda_{0}\right)$,

$$
\alpha_{k}+\kappa \alpha_{k-1} \leq \theta\left(\alpha_{k-1}+\kappa \alpha_{k-2}\right),
$$

where $\alpha_{k}=\left\|\left(m_{k}, \eta_{k}\right)-\left(m_{k}^{\prime}, \eta_{k}^{\prime}\right)\right\|$. As an immediate consequence we have the following corollary which will be used in Section 2.3. 
Corollary 2.4. Suppose that Assumptions 1.1-1.5 hold. Let $\epsilon_{0}, \lambda_{0} \in(0,1)$ be as in Lemma 2.3. Then with $\theta \in(0,1)$ as in Lemma 2.3 associated with a fixed choice of $\epsilon \leq \epsilon_{0}, \lambda \leq \lambda_{0}$, we have, for each $k \geq 2$

$$
\begin{aligned}
& \left\|\left(m_{k}, \eta_{k}\right)-\left(m_{k}^{\prime}, \eta_{k}^{\prime}\right)\right\|+\kappa\left\|\left(m_{k-1}, \eta_{k-1}\right)-\left(m_{k-1}^{\prime}, \eta_{k-1}^{\prime}\right)\right\| \\
\leq & \theta^{k-1}\left(\left\|\left(m_{1}, \eta_{1}\right)-\left(m_{1}^{\prime}, \eta_{1}^{\prime}\right)\right\|+\kappa\left\|\left(m_{0}, \eta_{0}\right)-\left(m_{0}^{\prime}, \eta_{0}^{\prime}\right)\right\|\right) .
\end{aligned}
$$

Suppose further that Assumption 1.8 holds and that $m_{0}$ has a density with respect to $\ell_{E}$ that is bounded by $M_{m_{0}}$. Then, for $k \geq 1$,

$$
\left\|\left(m_{k}, \eta_{k}\right)-\left(m_{k}^{\prime}, \eta_{k}^{\prime}\right)\right\| \leq \theta^{k-1}\left(2+\kappa+2 \lambda\left(M_{m_{0}}+M_{Q, Q_{0}}\right)\right)\left(\left\|\left(m_{0}, \eta_{0}\right)-\left(m_{0}^{\prime}, \eta_{0}^{\prime}\right)\right\|\right) .
$$

Proof. Equation (2.13) comes directly from (2.12). Next note that

$$
\left\|\eta_{1}-\eta_{1}^{\prime}\right\|_{T V} \leq\left\|m_{0}-m_{0}^{\prime}\right\|_{T V}+\left\|\eta_{0}-\eta_{0}^{\prime}\right\|_{T V} .
$$

Also, recalling (2.1),

$$
\begin{aligned}
\left\|m_{0} M^{\eta_{0}}-m_{0} M^{\eta_{0}^{\prime}}\right\|_{T V} & \leq 2 \int_{x, y \in E} m_{0}(\mathrm{~d} x) Q(x, \mathrm{~d} y) \lambda\left(\left|\eta_{0}(x)-\eta_{0}^{\prime}(x)\right|+\left|\eta_{0}(y)-\eta_{0}^{\prime}(y)\right|\right) \\
& \leq 2 \lambda\left(M_{m_{0}}+M_{Q, Q_{0}}\right)\left\|\eta_{0}-\eta_{0}^{\prime}\right\|_{T V}
\end{aligned}
$$

and

$$
\left\|m_{0} M^{\eta_{0}^{\prime}}-m_{0}^{\prime} M^{\eta_{0}^{\prime}}\right\|_{T V} \leq\left\|m_{0}-m_{0}^{\prime}\right\|_{T V}
$$

Combining these estimates

$$
\left\|\left(m_{1}, \eta_{1}\right)-\left(m_{1}^{\prime}, \eta_{1}^{\prime}\right)\right\| \leq\left(1+\sup \left(1,2 \lambda\left(M_{m_{0}}+M_{Q, Q_{0}}\right)\right)\right)\left\|\left(m_{0}, \eta_{0}\right)-\left(m_{0}^{\prime}, \eta_{0}^{\prime}\right)\right\| .
$$

Proof of Theorem 1.6. Take $\epsilon_{0}, \lambda_{0}$ as in Lemma 2.3 and fix $\epsilon \in\left(0, \epsilon_{0}\right)$ and $\lambda \in\left(0, \lambda_{0}\right)$. The uniqueness is immediate from Lemma 2.3. For existence, take any $\left(m_{0}, \eta_{0}\right) \in \mathcal{P}(E) \times \mathcal{P}^{*}\left(\mathbb{R}^{d}\right)$ and define recursively $\forall k \geq 1$, $\left(m_{k}, \eta_{k}\right)=\Phi\left(m_{k-1}, \eta_{k-1}\right)$. We have for all $k \geq 1, p \geq 1$, using the $\theta$ given by Lemma 2.3,

$$
\left\|\Phi^{k+p}\left(m_{0}, \eta_{0}\right)-\Phi^{k}\left(m_{0}, \eta_{0}\right)\right\| \leq 4 \theta^{k-1} .
$$

So the sequence $\left(m_{k}, \eta_{k}\right)$ is a Cauchy sequence in $\left(\mathcal{P}(E) \times \mathcal{P}^{*}\left(\mathbb{R}^{d}\right),\|\cdot\|\right)$ and thus it has a limit $\left(m_{\infty}, \eta_{\infty}\right)$. By taking $p \rightarrow+\infty$ in $(2.14)$, we see that $\left(m_{k}, \eta_{k}\right) \underset{k \rightarrow+\infty}{\longrightarrow}\left(m_{\infty}, \eta_{\infty}\right)$.

\subsection{Uniform convergence over the infinite time horizon}

In this subsection we will prove Theorem 1.9 and Corollary 1.10.

Proof of Theorem 1.9. Fix $\delta>0$. For $k \in \mathbb{N}$, we take $\Phi_{k+1}^{N}$ to be a (random) operator such that $\Phi_{k+1}^{N}\left(m_{k}^{N}, \eta_{k}^{N}\right)=$ $\left(m_{k+1}^{N}, \eta_{k+1}^{N}\right)$. By convention, we take $\Phi_{1}^{N}\left(m_{0}, \eta_{0}\right)=\left(m_{1}^{N}, \eta_{1}^{N}\right)$. Let us denote

$$
\text { for } 1 \leq j, \Phi_{1: j}^{N}\left(m_{0}, \eta_{0}\right)=\Phi_{j}^{N} \circ \Phi_{j-1}^{N} \circ \cdots \circ \Phi_{1}^{N}\left(m_{0}, \eta_{0}\right) \text { and for } j=0, \Phi_{1: j}^{N}\left(m_{0}, \eta_{0}\right)=\left(m_{0}, \eta_{0}\right) \text {. }
$$

Also, recall that, for $i<j, \Phi^{(j-i)}=\Phi \circ \cdots \circ \Phi(j-i$ times $)$. We set $\Phi^{(0)}=$ Id. Note that for $n \geq 1$,

$$
\left(m_{n}^{N}, \eta_{n}^{N}\right)-\left(m_{n}, \eta_{n}\right)=\sum_{k=1}^{n}\left[\Phi^{(n-k)} \circ \Phi_{k}^{N} \circ \Phi_{1: k-1}^{N}\left(m_{0}, \eta_{0}\right)-\Phi^{(n-k)} \circ \Phi \circ \Phi_{1: k-1}^{N}\left(m_{0}, \eta_{0}\right)\right] .
$$

We set $\forall k \in \mathbb{N}$,

$$
\begin{aligned}
\left(m_{k, k}^{N}, \eta_{k, k}^{N}\right)=\Phi_{1: k}^{N}\left(m_{0}, \eta_{0}\right), & \left(m_{k, k}, \eta_{k, k}\right)=\Phi \circ \Phi_{1: k-1}^{N}\left(m_{0}, \eta_{0}\right), \\
\left(m_{k, k+1}^{N}, \eta_{k, k+1}^{N}\right)=\Phi\left(m_{k, k}^{N}, \eta_{k, k}^{N}\right), & \left(m_{k, k+1}, \eta_{k, k+1}\right)=\Phi\left(m_{k, k}, \eta_{k, k}\right) .
\end{aligned}
$$


Then

$$
\begin{aligned}
\left(m_{n}^{N}, \eta_{n}^{N}\right)-\left(m_{n}, \eta_{n}\right)= & \sum_{k=2}^{n-1}\left(\Phi^{(n-k-1)}\left(m_{k, k+1}^{N}, \eta_{k, k+1}^{N}\right)-\Phi^{(n-k-1)}\left(m_{k, k+1}, \eta_{k, k+1}\right)\right) \\
& +\left(\left(m_{n, n}^{N}, \eta_{n, n}^{N}\right)-\left(m_{n, n}, \eta_{n, n}\right)\right) \\
& +\left(\Phi^{(n-1)}\left(m_{1}^{N}, \eta_{1}^{N}\right)-\Phi^{(n-1)}\left(m_{1}, \eta_{1}\right)\right) .
\end{aligned}
$$

Notice that

$$
\eta_{k, k}^{N}=\eta_{k, k} \text { for al } k>1 \text {. }
$$

Now fix a $k \in\{2, \ldots, n-1\}$. The signed measure $\eta_{k, k+1}^{N}-\eta_{k, k+1}$ has the following density

$$
\left(\eta_{k, k+1}^{N}-\eta_{k, k+1}\right)(y)=\epsilon \int_{x \in E}\left(m_{k}^{N}(\mathrm{~d} x)-m_{k, k}(\mathrm{~d} x)\right) P^{\prime}(x, y), y \in \mathbb{R}^{d} .
$$

From Assumption $1.7(2)$, for all $f \in \mathcal{B}_{1}\left(\mathbb{R}^{d}\right)$,

$$
x \in E \mapsto \int_{y \in \mathbb{R}^{d}} P^{\prime}(x, \mathrm{~d} y) f(y)
$$

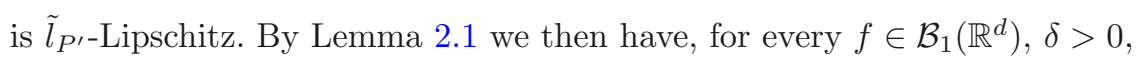

$\left|\eta_{k, k+1}^{N}(f)-\eta_{k, k+1}(f)\right|=\left|\epsilon \int_{x \in E}\left(m_{k, k}^{N}(\mathrm{~d} x)-m_{k, k}(\mathrm{~d} x)\right) \int_{y \in \mathbb{R}^{d}} P^{\prime}(x, \mathrm{~d} y) f(y)\right| \leq \epsilon\left(2 \delta+\sup _{g \in F_{1}^{\delta}}\left|\left\langle m_{k, k}^{N}-m_{k, k}, g\right\rangle\right|\right)$.

where $F_{1}^{\delta}=F_{1, \tilde{l}_{P^{\prime}}}^{\delta}(E)$. Now by Lemma A.5, with $C_{1}(\delta)=\left|F_{1}^{\delta}\right|$,

$$
\mathbb{E}\left(\left\|\eta_{k, k+1}^{N}-\eta_{k, k+1}\right\|_{T V}\right) \leq 2 \epsilon\left(\delta+\frac{C_{1}(\delta)}{\sqrt{N}}\right)
$$

Using (2.17) once again

$$
m_{k, k+1}^{N}-m_{k, k+1}=m_{k, k}^{N} M^{\eta_{k, k}}-m_{k, k} M^{\eta_{k, k}} .
$$

For any $f \in \mathcal{B}_{1}(E)$, we have

$$
\left\langle m_{k, k+1}^{N}-m_{k, k+1}, f\right\rangle=\left\langle m_{k, k}^{N}-m_{k, k}, M^{\eta_{k, k}} f\right\rangle .
$$

From Lemmas A.3 and A.4 in the Appendix we see that the function $x \in E \mapsto M^{\eta_{k, k}} f(x)$ is $\bar{l}_{Q, Q_{0}}$-Lipschitz and bounded by 1 where $\bar{l}_{Q, Q_{0}}=l_{Q, Q_{0}}\left(3+2 \lambda \bar{l}_{P, P^{\prime}}\right)$. So using Lemma 2.1 once again, we have, for $\delta>0$,

$$
\begin{aligned}
\mathbb{E}\left(\left\|m_{k, k+1}^{N}-m_{k, k+1}\right\|_{T V}\right) & \leq 2 \delta+\mathbb{E}\left(\sup _{g \in F_{2}^{\delta}}\left|\left\langle m_{k, k}^{N}-m_{k, k}, g\right\rangle\right|\right. \\
& \leq 2\left(\delta+\frac{C_{2}(\delta)}{\sqrt{N}}\right) .
\end{aligned}
$$

where $F_{2}^{\delta}=F_{1, \bar{l}_{Q, Q_{0}}}^{\delta}(E)$ and $C_{2}(\delta)=\left|F_{2}^{\delta}\right|$. We will now apply Corollary 2.4. Note that, from Assumption 1.8, for every $k, m_{k, k+1}^{N}$ has a density on $E$ with respect to $l_{E}$ that is bounded by $2 M_{Q, Q_{0}}$. This, in view of Corollary 2.4 , along with (2.19) and (2.21) yields $\forall k \in\{2, \ldots, n-2\}$,

$$
\mathbb{E}\left(\left\|\Phi^{n-k-1}\left(m_{k, k+1}^{N}, \eta_{k, k+1}^{N}\right)-\Phi^{n-k-1}\left(m_{k, k+1}, \eta_{k, k+1}\right)\right\|_{T V}\right) \leq \bar{C} \theta^{n-k-2}\left(4 \delta+\frac{C_{1}(\delta)+C_{2}(\delta)}{\sqrt{N}}\right),
$$


where $\bar{C}=\left(2+\kappa+6 \lambda M_{Q, Q_{0}}\right)$. Note that the above inequality holds trivially if $k=n-1$. For the term in the second line of (2.16), note that, for $n>1$,

$$
\sup _{f \in \mathcal{B}_{1}(E)} \mathbb{E}\left(\left|\left\langle m_{n, n}^{N}-m_{n, n}, f\right\rangle\right|\right) \leq \frac{2}{\sqrt{N}}, \eta_{n, n}^{N}=\eta_{n, n} .
$$

The norm of the term in the third line of (2.16), using Lemma 2.3, can be bounded by $4 \theta^{n-2}$. Combining these estimates, for all $n>1$

$$
\begin{aligned}
\sup _{f \in \mathcal{B}_{1}(E)} \mathbb{E}\left(\left|\left\langle m_{n}^{N}-m_{n}, f\right\rangle\right|+\left\|\eta_{n}^{N}-\eta_{n}\right\|_{T V}\right) & \leq \frac{2}{\sqrt{N}}+\sum_{k=2}^{n-1} \theta^{n-k-2} \bar{C}\left(4 \delta+\frac{C_{1}(\delta)+C_{2}(\delta)}{\sqrt{N}}\right)+4 \theta^{n-2} \\
& \leq \frac{2}{\sqrt{N}}+\bar{C}\left(4 \delta+\frac{C_{1}(\delta)+C_{2}(\delta)}{\sqrt{N}}\right) \frac{\theta^{-1}}{1-\theta}+4 \theta^{n-1} .
\end{aligned}
$$

The result now follows on combining the above estimate with Theorem 1.2.

Proof of Corollary 1.10. Fix $\delta>0$. From Theorem 1.6, there exist $\left(m_{\infty}, \eta_{\infty}\right) \in \mathcal{P}(E) \times \mathcal{P}^{*}\left(\mathbb{R}^{d}\right)$ and $n_{0}$ such that $\forall n \geq n_{0}$,

$$
\left\|\left(m_{n}, \eta_{n}\right)-\left(m_{\infty}, \eta_{\infty}\right)\right\|<\delta .
$$

From Theorem 1.9, there exist $N_{0}, n_{1} \in \mathbb{N}$ such that $\forall n \geq n_{1}, \forall N \geq N_{0}$,

$$
\sup _{f \in \mathcal{B}_{1}(E)} \mathbb{E}\left(\left|\left\langle m_{n}^{N}-m_{n}, f\right\rangle\right|+\left\|\eta_{n}-\eta_{n}^{N}\right\|_{T V}\right)<\delta .
$$

And so

$$
\begin{aligned}
& \limsup _{n \rightarrow+\infty} \limsup _{N \rightarrow+\infty} \sup _{f \in \mathcal{B}_{1}(E)} \mathbb{E}\left(\left|\left\langle m_{n}^{N}-m_{\infty}, f\right\rangle\right|+\left\|\eta_{n}^{N}-\eta_{\infty}\right\|_{T V}\right)<2 \delta, \\
& \limsup _{N \rightarrow+\infty} \limsup _{n \rightarrow+\infty} \sup _{f \in \mathcal{B}_{1}(E)} \mathbb{E}\left(\left|\left\langle m_{n}^{N}-m_{\infty}, f\right\rangle\right|+\left\|\eta_{n}^{N}-\eta_{\infty}\right\|_{T V}\right)<2 \delta .
\end{aligned}
$$

\subsection{Proof of Theorem 1.12}

In this subsection we will take Assumption 1.11 to hold. Recall that under Assumption 1.11, we have that Assumptions 1.1, 1.5 and 1.7 hold automatically.

Proof of Theorem 1.12. We proceed recursively. Since $\tilde{\eta}_{0}^{N}=\eta_{0}$, we have using Lemma A.5 that (1.13) holds for $k=0$. Suppose now that (1.13) holds for some $k \in \mathbb{N}_{0}$. Fix $\delta>0$. We have $\forall y \in E$,

$$
\tilde{\eta}_{k+1}^{N}(y)=(1-\epsilon)\left(P \star S^{N}\left(\tilde{\eta}_{k}^{N}\right)\right)(y)+\epsilon\left(P^{\prime} \star \tilde{m}_{k}^{N}\right)(y) .
$$

By Assumption 1.1 and Lemma 2.1, we can write, for $\delta>0$,

$$
\begin{aligned}
\mathbb{E}\left(\sup _{y \in \mathbb{R}^{d}}\left|\left(P^{\prime} \star \tilde{m}_{k}^{N}\right)(y)-\left(P^{\prime} \star m_{k}\right)(y)\right|\right) & =\mathbb{E}\left(\sup _{y \in \mathbb{R}^{d}}\left|\left\langle\tilde{m}_{k}^{N}-m_{k}, P^{\prime}(., y)\right\rangle\right|\right) \\
& \leq \mathbb{E}\left(\sup _{g \in F_{3}^{\delta}}\left|\left\langle\tilde{m}_{k}^{N}-m_{k}, g\right\rangle\right|+2 \delta\right) \\
& \leq \sum_{g \in F_{3}^{\delta}} \mathbb{E}\left(\left|\left\langle\tilde{m}_{k}^{N}-m_{k}, g\right\rangle\right|\right)+2 \delta,
\end{aligned}
$$

where $F_{3}^{\delta}=F_{M_{P^{\prime}}, l_{P^{\prime}}}^{\delta}(E)$. By Lemma A.7, there exists $K(\delta)$ compact such that

$$
\eta_{k}\left(K(\delta)^{c}\right)<\delta, \mathbb{E}\left(\tilde{\eta}_{k}^{N}\left(K(\delta)^{c}\right)\right)<\delta, \forall k, N
$$


Using Assumption 1.5, 1.7(1) and Lemma 2.1, we can write,

$$
\begin{aligned}
\mathbb{E}\left(\sup _{y \in \mathbb{R}^{d}} \mid\left(S^{N}\left(\tilde{\eta}_{k}^{N}\right) P(y)-\left(\tilde{\eta}_{k}^{N} P\right)(y) \mid\right)\right. & \leq \mathbb{E}\left(\sup _{y \in \mathbb{R}^{d}}\left|\left\langle S^{N}\left(\tilde{\eta}_{k}^{N}\right)-\tilde{\eta}_{k}^{N}, P(., y) \mathbf{1}_{K(\delta)}(.)\right\rangle\right|+\left|\left\langle S^{N}\left(\tilde{\eta}_{k}^{N}\right)-\tilde{\eta}_{k}^{N}, P(., y) \mathbf{1}_{K(\delta)^{c}}(.)\right\rangle\right|\right) \\
& \leq \sum_{g \in F_{4}^{\delta}} \mathbb{E}\left(\left|\left\langle S^{N}\left(\tilde{\eta}_{k}^{N}\right)-\tilde{\eta}_{k}^{N}, g \mathbf{1}_{K(\delta)}(.)\right\rangle\right|\right)+2 \delta\left(1+M_{P}\right) \\
& \leq 2\left(\frac{C_{4}(\delta) M_{P}}{\sqrt{N}}+\delta\left(1+M_{P}\right)\right)
\end{aligned}
$$

where $F_{4}^{\delta}=F_{M_{P}, l_{P}}^{\delta}(K(\delta)), C_{4}(\delta)=\left|F_{4}^{\delta}\right|$ and the last inequality is a consequence of Lemma A.5.

In a similar manner

$$
\mathbb{E}\left(\sup _{y \in \mathbb{R}^{d}}\left|\tilde{\eta}_{k}^{N} P(y)-\eta_{k} P(y)\right|\right) \leq \sum_{g \in F_{4}^{\delta}} \mathbb{E}\left(\left|\left\langle\tilde{\eta}_{k}^{N}-\eta_{k}, g \mathbf{1}_{K(\delta)}(.)\right\rangle\right|\right)+2 \delta\left(1+M_{P}\right) .
$$

And so, by (2.22)-(2.25),

$$
\begin{aligned}
\mathbb{E}\left(\left\|\tilde{\eta}_{k+1}^{N}-\eta_{k+1}\right\|_{\infty}\right) \leq & \left(\frac{2 C_{4}(\delta) M_{P}}{\sqrt{N}}+6 \delta\left(1+M_{P}\right)\right)+\sum_{g \in F_{3}^{\delta}} \mathbb{E}\left(\left|\left\langle\tilde{m}_{k}^{N}-m_{k}, g\right\rangle\right|\right) \\
& +2 \sum_{g \in F_{4}^{\delta}} \mathbb{E}\left(\left|\left\langle\tilde{\eta}_{k}^{N}-\eta_{k}, g \mathbf{1}_{K(\delta)}\right\rangle\right|\right) .
\end{aligned}
$$

Recalling that (1.13) is assumed for $k$, we have that, as $N \rightarrow \infty$,

$$
\mathbb{E}\left(\left\|\tilde{\eta}_{k+1}^{N}-\eta_{k+1}\right\|_{\infty}\right) \rightarrow 0
$$

An application of Scheffe's theorem now shows that, as $N \rightarrow \infty$,

$$
\mathbb{E}\left(\left\|\tilde{\eta}_{k+1}^{N}-\eta_{k+1}\right\|_{T V}\right) \rightarrow 0
$$

Next, for any $f \in \mathcal{B}_{1}(E)$, we have

$$
\begin{aligned}
\mathbb{E}\left(\left|\left\langle\tilde{m}_{k+1}^{N}-m_{k+1}, f\right\rangle\right|\right) \leq & \mathbb{E}\left(\left|\tilde{m}_{k+1}^{N}-\tilde{m}_{k}^{N} M^{\tilde{\eta}_{k}^{N}}, f\right\rangle \mid\right) \\
& +\mathbb{E}\left(\left|\left\langle\tilde{m}_{k}^{N} M^{\tilde{\eta}_{k}^{N}}-\tilde{m}_{k}^{N} M^{\eta_{k}}, f\right\rangle\right|\right)+\mathbb{E}\left(\left|\left\langle\tilde{m}_{k}^{N} M^{\eta_{k}}-m_{k} M^{\eta_{k}}, f\right\rangle\right|\right) \\
\leq & \frac{2}{\sqrt{N}}+4 \lambda \mathbb{E}\left(\left\|\tilde{\eta}_{k}^{N}-\eta_{k}\right\|_{\infty}\right)+\mathbb{E}\left(\left|\left\langle\tilde{m}_{k}^{N}-m_{k}, M^{\eta_{k}} f\right\rangle\right|\right),
\end{aligned}
$$

where the last inequality uses (2.1) and Lemma A.5. Once again using the recurrence assumption, we now have that

$$
\sup _{f \in \mathcal{B}_{1}(E)} \mathbb{E}\left(\left|\left\langle\tilde{m}_{k+1}^{N}-m_{k+1}, f\right\rangle\right|\right) \underset{N \rightarrow+\infty}{\longrightarrow} 0
$$

Thus we have proved that (1.13) holds for $k+1$. The result follows.

\subsection{Proof of Theorem $\mathbf{1 . 1 3}$}

Proof. For $k \in \mathbb{N}$, we take $\bar{\Phi}_{k+1}^{N}$ to be a (random) operator such that $\bar{\Phi}_{k+1}^{N}\left(\tilde{m}_{k}^{N}, \tilde{\eta}_{k}^{N}\right)=\left(\tilde{m}_{k+1}^{N}, \tilde{\eta}_{k+1}^{N}\right)$. By convention we take $\bar{\Phi}_{1}^{N}\left(m_{0}, \eta_{0}\right)=\left(\tilde{m}_{1}^{N}, \tilde{\eta}_{1}^{N}\right)$. Following the Proof of Theorem 1.9, we define

for $1 \leq j, \bar{\Phi}_{1: j}^{N}\left(m_{0}, \eta_{0}\right)=\bar{\Phi}_{j}^{N} \circ \bar{\Phi}_{j-1}^{N} \circ \cdots \circ \bar{\Phi}_{1}^{N}\left(m_{0}, \eta_{0}\right)$, and for $j=0, \bar{\Phi}_{1: j}^{N}\left(m_{0}, \eta_{0}\right)=\left(m_{0}, \eta_{0}\right)$. 
We define $\forall k \in \mathbb{N}$,

$$
\begin{aligned}
\left(m_{k, k}^{N}, \eta_{k, k}^{N}\right) & =\bar{\Phi}_{1: k}^{N}\left(m_{0}, \eta_{0}\right),\left(m_{k, k}, \eta_{k, k}\right)=\Phi \circ \bar{\Phi}_{1: k-1}^{N}\left(m_{0}, \eta_{0}\right), \\
\left(m_{k, k+1}^{N}, \eta_{k, k+1}^{N}\right) & =\Phi\left(m_{k, k}^{N}, \eta_{k, k}^{N}\right),\left(m_{k, k+1}, \eta_{k, k+1}\right)=\Phi\left(m_{k, k}, \eta_{k, k}\right) .
\end{aligned}
$$

We use the same symbols as in the Proof of Theorem 1.9 in order to keep notations simple. We have the following telescopic decomposition

$$
\left(\tilde{m}_{n}^{N}, \tilde{\eta}_{n}^{N}\right)-\left(m_{n}, \eta_{n}\right)=\sum_{k=1}^{n}\left[\Phi^{(n-k)} \circ \bar{\Phi}_{k}^{N} \circ \bar{\Phi}_{1: k-1}^{N}\left(m_{0}, \eta_{0}\right)-\Phi^{(n-k)} \circ \Phi \circ \bar{\Phi}_{1: k-1}^{N}\left(m_{0}, \eta_{0}\right)\right] .
$$

The proof is very similar to the Proof of Theorem 1.9, except that now $\eta_{k, k}^{N} \neq \eta_{k, k}$. The strategy remain the same; we want to bound the 'local error term' $\Phi^{(2)} \circ \bar{\Phi}_{1: k-1}^{N}-\Phi \circ \bar{\Phi}_{1: k}^{N}$ in total variation and then use the contraction property of Lemma 2.3 to bound the telescopic sum uniformly in $N$. Fix $(\epsilon, \lambda) \in(0, \infty)$ such that $\epsilon \leq \epsilon_{0}$ and $\lambda \leq \lambda_{0}$, where $\epsilon_{0}, \lambda_{0}$ are as in Lemma 2.3. Let $\theta=\theta(\epsilon, \lambda)$. Also, fix $\delta>0$. Consider a $k \in\{2, \ldots, n-1\}$. Note that

Therefore, from (2.24)

$$
\eta_{k, k}^{N}-\eta_{k, k}=(1-\epsilon)\left(S^{N}\left(\tilde{\eta}_{k-1}^{N}\right)-\tilde{\eta}_{k-1}^{N}\right) P .
$$

$$
\mathbb{E}\left(\left\|\eta_{k, k}^{N}-\eta_{k, k}\right\|_{\infty}\right) \leq 2\left(\frac{C_{4}(\delta) M_{P}}{\sqrt{N}}+\delta\left(1+M_{P}\right)\right) .
$$

In the same way as (2.21) in the Proof of Theorem 1.9 (see also (2.20)), we get

$$
\mathbb{E}\left(\left\|m_{k, k}^{N} M^{\eta_{k, k}}-m_{k, k} M^{\eta_{k, k}}\right\|_{T V}\right) \leq 2\left(\delta+\frac{C_{2}(\delta)}{\sqrt{N}}\right) .
$$

Also from (2.1) and Lemma 2.2, we have $\forall f \in \mathcal{B}_{1}(E)$

$$
\mid\left\langle m_{k, k}^{N} M^{\eta_{k, k}^{N}}-m_{k, k}^{N} M^{\eta_{k, k}}, f>\right| \leq 4\left\|\eta_{k, k}^{N}-\eta_{k, k}\right\|_{\infty} .
$$

Equations (2.29)-(2.31) yield

$$
\mathbb{E}\left(\left\|m_{k, k+1}^{N}-m_{k, k+1}\right\|_{T V}\right) \leq \frac{8 C_{4}(\delta) M_{P}+2 C_{2}(\delta)}{\sqrt{N}}+\delta\left(10+8 M_{P}\right) .
$$

Next, with $K(\delta)$ as in the Proof of Theorem 1.12, $\forall f \in \mathcal{B}_{1}\left(\mathbb{R}^{d}\right)$

$$
\begin{aligned}
\left|\eta_{k, k}^{N}(f)-\eta_{k, k}(f)\right| & =\left|(1-\epsilon) \int_{x \in \mathbb{R}^{d}, y \in \mathbb{R}^{d}} P(x, y) f(y) \mathrm{d} y\left(S^{N}\left(\tilde{\eta}_{k-1}^{N}\right)-\tilde{\eta}_{k-1}^{N}\right)(\mathrm{d} x)\right| \\
& \leq\left|\left\langle S^{N}\left(\tilde{\eta}_{k-1}^{N}\right)-\tilde{\eta}_{k-1}^{N}, P f(\cdot) \mathbf{1}_{K(\delta)}(\cdot)\right\rangle\right|+\left|S^{N}\left(\tilde{\eta}_{k-1}^{N}\right)\left(K(\delta)^{c}\right)\right|+\left|\tilde{\eta}_{k-1}^{N}\left(K(\delta)^{c}\right)\right| .
\end{aligned}
$$

Also, using the Gaussian property of the kernel $P$, it follows that $P f$ is Lipschitz on $K(\delta)$, uniformly in $f \in \mathcal{B}_{1}\left(\mathbb{R}^{d}\right)$. Denote the uniform bound on the Lipschitz norm by $l_{K(\delta)}$. Then

$$
\left|\left\langle S^{N}\left(\tilde{\eta}_{k}^{N}\right)-\tilde{\eta}_{k}^{N}, \operatorname{Pf} \mathbf{1}_{K(\delta)}\right\rangle\right| \leq \max _{g \in F_{6}^{\delta}}\left|\left\langle S^{N}\left(\tilde{\eta}_{k}^{N}\right)-\tilde{\eta}_{k}^{N}, g \mathbf{1}_{K(\delta)}\right\rangle\right|+2 \delta,
$$

where $F_{6}^{\delta}=F_{1, l_{K(\delta)}}^{\delta}(K(\delta))$. Thus

$$
\mathbb{E}\left|\eta_{k, k}^{N}(f)-\eta_{k, k}(f)\right| \leq \mathbb{E} \max _{g \in F_{6}^{\delta}}\left|\left\langle S^{N}\left(\tilde{\eta}_{k-1}^{N}\right)-\tilde{\eta}_{k-1}^{N}, g \mathbf{1}_{K(\delta)}\right\rangle\right|+4 \delta
$$


Next, for all $f \in \mathcal{B}_{1}\left(\mathbb{R}^{d}\right)$

$$
\begin{aligned}
\mathbb{E}\left|\eta_{k, k+1}^{N}(f)-\eta_{k, k+1}(f)\right| & =\mathbb{E}\left|\left\langle(1-\epsilon)\left(\eta_{k, k}^{N}-\eta_{k, k}\right) P+\epsilon\left(m_{k, k}^{N}-m_{k, k}\right) P^{\prime}, f\right\rangle\right| \\
& \leq \mathbb{E}\left|\left\langle\eta_{k, k}^{N}-\eta_{k, k}, P f\right\rangle\right|+\mathbb{E}\left|\left\langle m_{k, k}^{N} P^{\prime}-m_{k, k} P^{\prime}, f\right\rangle\right| \\
& \leq \mathbb{E}\left(\max _{g \in F_{6}^{\delta}}\left|\left\langle S^{N}\left(\tilde{\eta}_{k-1}^{N}\right)-\tilde{\eta}_{k-1}^{N}, g \mathbf{1}_{K(\delta)}\right\rangle\right|\right)+4 \delta+\mathbb{E}\left\|m_{k, k}^{N} P^{\prime}-m_{k, k} P^{\prime}\right\|_{T V} .
\end{aligned}
$$

Using $C_{1}(\delta)$ introduced in the Proof of Theorem 1.9, we have

$$
\mathbb{E}\left(\left\|m_{k, k}^{N} P^{\prime}-m_{k, k} P^{\prime}\right\|_{T V}\right) \leq 2\left(\delta+\frac{C_{1}(\delta)}{\sqrt{N}}\right) .
$$

We then get

$$
\mathbb{E}\left(\left\|\eta_{k, k+1}^{N}-\eta_{k, k+1}\right\|_{T V}\right) \leq 2\left(3 \delta+\frac{C_{1}(\delta)+C_{6}(\delta)}{\sqrt{N}}\right)
$$

where $C_{6}(\delta)=\left|F_{6}^{\delta}\right|$. Also, by $(2.33)$

$$
\mathbb{E}\left(\left\|\eta_{n, n}^{N}-\eta_{n, n}\right\|_{T V}\right) \leq 2\left(2 \delta+\frac{C_{6}(\delta)}{\sqrt{N}}\right),
$$

and using the definitions of $m_{n, n}^{N}$ and $m_{n, n}$, we get from Lemma A.5

$$
\sup _{f \in \mathcal{B}_{1}(E)} \mathbb{E}\left(\left|\left\langle m_{n, n}^{N}-m_{n, n}, f\right\rangle\right|\right) \leq \frac{2}{\sqrt{N}}
$$

Thus as in the Proof of Theorem 1.9 we get, for $n>1$,

$$
\begin{aligned}
\sup _{f \in \mathcal{B}_{1}(E)} \mathbb{E}\left(\left|\left\langle\tilde{m}_{n}^{N}-m_{n}, f\right\rangle\right|+\left\|\tilde{\eta}_{n}^{N}-\eta_{n}\right\|_{T V}\right) & \\
& \leq \frac{2}{\sqrt{N}}+2\left(2 \delta+\frac{C_{6}(\delta)}{\sqrt{N}}\right)+\bar{C}\left(\delta\left(3+M_{P}\right)+\frac{2 C_{1}(\delta)+2 C_{2}(\delta)+2 C_{6}(\delta)+8 C_{4}(\delta)}{\sqrt{N}}\right) \\
& \times \frac{\theta^{-2}}{1-\theta}+4 \theta^{n-1}
\end{aligned}
$$

\section{Appendix. Auxiliary Results}

Lemma A.1. Let $M$ be a transition probability kernel on $E$ such that for some $\epsilon \in(0,1)$ and $\ell_{1} \in \mathcal{P}(E)$,

$$
M(x, A) \geq \epsilon \ell_{1}(A), \forall A \in \mathcal{B}(E), x \in E .
$$

Then, for all $\mu_{1}, \mu_{2} \in \mathcal{P}(E)$,

$$
\left\|\mu_{1} M-\mu_{2} M\right\|_{T V} \leq(1-\epsilon)\left\|\mu_{1}-\mu_{2}\right\|_{T V} .
$$

Proof. This result comes from Dobrushin's theorem and the proof of this theorem can be found in [1], pages 183 and 192 (see useful definitions on p. 181). Dobrushin's theorem can also be found in [9], page 70, with the corresponding proof in [10], page 332 .

Lemma A.2. Suppose that Assumption 1.4 holds. Then, for any $m, m^{\prime} \in \mathcal{P}\left(\mathbb{R}^{d}\right)$ and $\eta \in \mathcal{P}^{*}\left(\mathbb{R}^{d}\right)$,

$$
\left\|m M^{\eta}-m^{\prime} M^{\eta}\right\|_{T V} \leq\left(1-\epsilon_{Q} \mathrm{e}^{-\lambda o s c(\eta)}\right)\left\|m-m^{\prime}\right\| .
$$


Proof. The proof can be found in [1], page 195. Since this book is written in French, we give a quick proof. Note that $\forall x, y \in E$,

$$
M^{\eta}(x, \mathrm{~d} y)=Q(x, \mathrm{~d} y) \mathrm{e}^{-\lambda(\eta(x)-\eta(y))_{+}}+Q_{0}(x, \mathrm{~d} y)\left(1-\int_{z \in E} Q(x, \mathrm{~d} z) \mathrm{e}^{-\lambda(\eta(x)-\eta(z))_{+}}\right) .
$$

Then by Assumption 1.4

$$
M^{\eta}(x, \mathrm{~d} y) \geq \epsilon_{Q} \mathrm{e}^{-\lambda \mathrm{osc}(\eta)} l_{1}(\mathrm{~d} y) .
$$

The result now follows from Lemma A.1.

Lemma A.3. Let $\eta^{\prime} \in \mathcal{P}\left(\mathbb{R}^{d}\right), m \in \mathcal{P}(E)$, and let $\eta=\eta^{\prime} R_{m}$. Suppose that Assumption 1.7 (3) holds. Then, $\eta$ has a $\bar{l}_{P, P^{\prime}}-$ Lipschitz density.

Proof. Under Assumption 1.7 (3), the density of $\eta$ is

$$
x \mapsto(1-\epsilon) \int_{y \in \mathbb{R}^{d}} \eta^{\prime}(\mathrm{d} y) P(y, x)+\epsilon \int_{y \in \mathbb{R}^{d}} m(\mathrm{~d} y) P(y, x) .
$$

And for $x, x^{\prime} \in \mathbb{R}^{d}$,

$$
\begin{aligned}
\left|\eta(x)-\eta\left(x^{\prime}\right)\right| & \leq(1-\epsilon) \int_{y \in \mathbb{R}^{d}} \eta^{\prime}(\mathrm{d} y) \bar{l}_{P, P^{\prime}}\left|x-x^{\prime}\right|+\epsilon \int_{y \in \mathbb{R}^{d}} m(\mathrm{~d} y) \bar{l}_{P, P^{\prime}}\left|x-x^{\prime}\right| \\
& =\bar{l}_{P, P^{\prime}}\left|x-x^{\prime}\right| .
\end{aligned}
$$

Lemma A.4. Take $\eta \in \mathcal{P}\left(\mathbb{R}^{d}\right)$ having a $l_{\eta}$-Lipschitz density and let $f \in \mathcal{B}_{1}\left(\mathbb{R}^{d}\right)$. Under Assumption 1.8, the function $x \in E \mapsto M^{\eta} f(x)$ is $\bar{l}_{Q, Q_{0}}$-Lipschitz with

$$
\bar{l}_{Q, Q_{0}}=l_{Q, Q_{0}}\left(3+2 \lambda l_{\eta}\right) .
$$

Proof. Note that for $x \in E$

$$
M^{\eta} f(x)=\int_{y \in E} Q(x, \mathrm{~d} y) \mathrm{e}^{-\lambda(\eta(x)-\eta(y))_{+}} f(y)+\int_{y \in E} Q_{0}(x, \mathrm{~d} y) f(y) \times\left(1-\int_{z \in E} Q(x, \mathrm{~d} z)\left(1-\mathrm{e}^{-\lambda(\eta(x)-\eta(z))_{+}}\right) .\right.
$$

So for $x, x^{\prime} \in E$, using Assumption 1.8

$$
\begin{aligned}
\left|M^{\eta} f(x)-M^{\eta} f\left(x^{\prime}\right)\right| \leq & l_{Q, Q_{0}}\left|x-x^{\prime}\right|+\int_{y \in E} Q\left(x^{\prime}, \mathrm{d} y\right)\left|\mathrm{e}^{-\lambda(\eta(x)-\eta(y))_{+}}-\mathrm{e}^{-\lambda\left(\eta\left(x^{\prime}\right)-\eta(y)\right)_{+}}\right| \\
& +\left|\int_{y \in E}\left(Q_{0}(x, \mathrm{~d} y)-Q_{0}\left(x^{\prime}, \mathrm{d} y\right)\right) f(y)\right| \times\left(1-\int_{z \in E} Q(x, \mathrm{~d} z)\left(1-\mathrm{e}^{-\lambda(\eta(x)-\eta(z))_{+}}\right)\right. \\
& +\int_{y \in E} Q_{0}\left(x^{\prime}, \mathrm{d} y\right) \times\left|\int_{z \in E} Q(x, \mathrm{~d} z) \mathrm{e}^{-\lambda(\eta(x)-\eta(z))_{+}}-Q\left(x^{\prime}, \mathrm{d} z\right) \mathrm{e}^{-\lambda\left(\eta\left(x^{\prime}\right)-\eta(z)\right)_{+}}\right| \\
\leq & l_{Q, Q_{0}}\left|x-x^{\prime}\right|+\int_{y \in E} Q\left(x^{\prime}, \mathrm{d} y\right) \lambda\left|\eta(x)-\eta\left(x^{\prime}\right)\right|+l_{Q, Q_{0}}\left|x-x^{\prime}\right| \\
& +l_{Q, Q_{0}}\left|x-x^{\prime}\right|+\int_{y \in E} Q\left(x^{\prime}, \mathrm{d} y\right) \lambda\left|\eta(x)-\eta\left(x^{\prime}\right)\right| \\
\leq & l_{Q, Q_{0}}\left|x-x^{\prime}\right|\left(3+2 \lambda l_{\eta}\right) .
\end{aligned}
$$




\section{Lemma A.5.}

(1) Let $E_{0}$ be a closed subset of $\mathbb{R}^{d}$ and let $\mu \in \mathcal{P}\left(E_{0}\right)$. Then for all $f \in \mathcal{B}_{1}\left(E_{0}\right)$

$$
\mathbb{E}\left(\left|\left\langle S^{N}(\mu)-\mu, f\right\rangle\right|\right) \leq \frac{2}{\sqrt{N}} .
$$

(2) Let $G$ be a transition probability kernel on a closed subset $E_{0}$ of $\mathbb{R}^{d}$. Let $x_{1}, \ldots, x_{N} \in E_{0}$ for some $N \in \mathbb{N}$ and let $\xi_{1}, \ldots, \xi_{N}$ be mutually independent random variables distributed as $\delta_{x_{1}} G, \ldots, \delta_{x_{N}} G$. Define $m_{0}^{N}=$ $\frac{1}{N} \sum_{i=1}^{N} \delta_{x_{i}}$ and $m_{1}^{N}=\frac{1}{N} \sum_{i=1}^{N} \delta_{\xi_{i}}$. Then for all $f \in \mathcal{B}_{1}\left(E_{0}\right)$

$$
\mathbb{E}\left(\left|\left\langle m_{1}^{N}-m_{0}^{N} G, f\right\rangle\right|\right) \leq \frac{2}{\sqrt{N}}
$$

Proof. We will only show (2). Proof of (1) is similar. Fix $f \in \mathcal{B}_{1}\left(E_{0}\right)$. Then

$$
\begin{aligned}
\mathbb{E}\left(\left|\left\langle m_{1}^{N}-m_{0}^{N} G, f\right\rangle\right|\right)= & \mathbb{E}\left|\frac{1}{N} \sum_{i=1}^{N} f\left(\xi_{i}\right)-\delta_{x_{i}} G(f)\right| \\
& \leq\left(\mathbb{E}\left(\frac{1}{N} \sum_{i=1}^{N} f\left(\xi_{i}\right)-\delta_{x_{i}} G(f)\right)^{2}\right)^{1 / 2} \\
& =\left(\mathbb{E} \frac{1}{N^{2}} \sum_{i=1}^{N}\left(f\left(\xi_{i}\right)-\delta_{x_{i}} G(f)\right)^{2}\right)^{1 / 2} \\
& \leq \frac{2}{\sqrt{N}} .
\end{aligned}
$$

Lemma A.6. Suppose $P, P^{\prime}$ satisfy Assumption 1.11, then they satisfy Assumption 1.5(1).

Proof. Let $m_{0}, m_{0}^{\prime} \in \mathcal{P}(E)$. We can write

$$
\left\{\begin{array}{l}
m_{0}=m+\tilde{m} \\
m_{0}^{\prime}=m+\tilde{m}^{\prime}
\end{array}\right.
$$

where $m, \tilde{m}, \tilde{m}^{\prime}$ are finite measures on $E$ and $\left\|m_{0}-m_{0}^{\prime}\right\|_{T V}=\left\|\tilde{m}-\tilde{m}^{\prime}\right\|_{T V}=\|\tilde{m}\|+\left\|\tilde{m}^{\prime}\right\|_{T V}$. We set

$$
\left\{\begin{array}{l}
P_{E}^{\prime}\left(x, \mathrm{~d} x^{\prime}\right)=P^{\prime}\left(x, \mathrm{~d} x^{\prime}\right) \mathbf{1}_{x^{\prime} \in E} \\
P_{E^{c}}^{\prime}\left(x, \mathrm{~d} x^{\prime}\right)=P^{\prime}\left(x, \mathrm{~d} x^{\prime}\right) \mathbf{1}_{x^{\prime} \in E^{c}} .
\end{array}\right.
$$

Since $E$ is compact, we can find $p \in \mathcal{P}(E)$ and $\delta>0$ such that

$$
P_{E}^{\prime}\left(x, \mathrm{~d} x^{\prime}\right)=\delta p\left(\mathrm{~d} x^{\prime}\right)+M\left(x, \mathrm{~d} x^{\prime}\right), \forall x \in E .
$$

where $M$ is some nonnegative kernel. Then

$$
\begin{aligned}
\left\|m_{0} P^{\prime}-m_{0}^{\prime} P^{\prime}\right\|_{T V} & =\left\|\tilde{m} P_{E}^{\prime}-\tilde{m}^{\prime} P_{E}^{\prime}\right\|_{T V}+\left\|\tilde{m} P_{E^{c}}^{\prime}-\tilde{m}^{\prime} P_{E}^{\prime}\right\|_{T V} \\
& \leq\left\|\tilde{m} M-\tilde{m}^{\prime} M\right\|_{T V}+\tilde{m} P^{\prime}\left(E^{c}\right)+\tilde{m}^{\prime} P^{\prime}\left(E^{c}\right) \\
& \leq \tilde{m} M(E)+\tilde{m}^{\prime} M(E)+\tilde{m} P^{\prime}\left(E^{c}\right)+\tilde{m}^{\prime} P^{\prime}\left(E^{c}\right) .
\end{aligned}
$$

From (A.1) we have

$$
\begin{aligned}
\tilde{m} M(E)+\tilde{m} P^{\prime}\left(E^{c}\right) & =\tilde{m} P^{\prime}(E)-\delta \tilde{m}(E)+\tilde{m} P^{\prime}\left(E^{c}\right) \\
& \leq(1-\delta) \tilde{m} P^{\prime}(E)+\tilde{m} P^{\prime}\left(E^{c}\right) \\
& =(1-\delta) \tilde{m} P^{\prime}(E)+\left(\tilde{m}(E)-\tilde{m} P^{\prime}(E)\right) .
\end{aligned}
$$


Since $E$ has a non-empty interior, we can find $\alpha>0$ such that $\forall \bar{m} \in \mathcal{P}(E), \bar{m} P^{\prime}(E) \geq \alpha$. In particular we have $\tilde{m} P^{\prime}(E) \geq \alpha \tilde{m}(E)$. Using this property in the above display

$$
\begin{aligned}
\tilde{m} M(E)+\tilde{m} P^{\prime}\left(E^{c}\right) & \leq(1-\delta) \alpha \tilde{m}(E)+(1-\alpha) \tilde{m}(E) \\
& =(1-\alpha \delta) \tilde{m}(E),
\end{aligned}
$$

Also, the same formula holds with $\tilde{m}$ replaced by $\tilde{m}^{\prime}$. So

$$
\begin{aligned}
\left\|m_{0} P^{\prime}-m_{0}^{\prime} P^{\prime}\right\|_{T V} & \leq(1-\alpha \delta)\left(\tilde{m}(E)+\tilde{m}^{\prime}(E)\right) \\
& =(1-\alpha \delta)\left\|m_{0}-m_{0}^{\prime}\right\|_{T V} .
\end{aligned}
$$

Lemma A.7. Suppose that Assumption $1.7(1.7)$ holds. Fix $\left(m_{0}, \eta_{0}\right) \in \mathcal{P}(E) \times \mathcal{P}^{*}\left(\mathbb{R}^{d}\right)$. Let $\eta_{k}$ be as defined in (1.10) and let $\tilde{\eta}_{k}^{N}$ be as defined in (1.12). Then, for any $\delta>0, \exists$ a compact subset of $\mathbb{R}^{d}, K(\delta)$, such that $\forall k, \forall N$,

$$
\eta_{k}\left(K(\delta)^{c}\right)<\delta, \mathbb{E}\left(\tilde{\eta}_{k}^{N}\left(K(\delta)^{c}\right)<\delta .\right.
$$

Proof. We can prove by recurrence that $\forall n$,

$$
\eta_{n+1}=\sum_{k=0}^{n}\left[\epsilon(1-\epsilon)^{k} m_{n-k} P^{\prime} P^{k}\right]+(1-\epsilon)^{n+1} \eta_{0} P^{n+1} .
$$

Fix $\tilde{\delta}>0$. Let $k_{0}$ such that $\sum_{k \geq k_{0}+1}(1-\epsilon)^{k}<\tilde{\delta}$. Since $E$ is compact, we can find a finite family $\left(x_{j}\right)_{j \in J}$ of elements of $E$ such that $\forall y \in E, \exists j \in J$ such that $\left|y-x_{j}\right|<\tilde{\delta}$. We can partition: $E=\sqcup_{j \in J} E_{j}$ such that $\forall j$ and $\forall y \in E_{j},\left|y-x_{j}\right|<\tilde{\delta}$. For each $j \in J$, we can find $K_{j}$, a compact subset of $\mathbb{R}^{d}$, such that $\delta_{x_{j}} P^{\prime}\left(K_{j}^{c}\right)<\tilde{\delta}, \delta_{x_{j}} P^{\prime} P\left(K_{j}^{c}\right)<\tilde{\delta}, \ldots, \delta_{x_{j}} P^{\prime} P^{k_{0}}\left(K_{j}^{c}\right)<\tilde{\delta}$. Clearly, the set $K=\cup_{j \in J} K_{j}$ is compact. Also, $\forall k \leq k_{0}, \forall r$

$$
\begin{aligned}
m_{r} P^{\prime} P^{k}\left(K^{c}\right)= & \int_{y \in E} m_{r}(\mathrm{~d} y)\left(P^{\prime} P^{k}\right)\left(y, K^{c}\right) \\
= & \sum_{j \in J} \int_{y \in E_{j}} m_{r}(\mathrm{~d} y)\left(P^{\prime} P^{k}\right)\left(y, K^{c}\right) \\
= & \sum_{j \in J} \int_{y \in E_{j}} m_{r}(\mathrm{~d} y)\left(P^{\prime} P^{k}\right)\left(x_{j}, K^{c}\right) \\
& +\sum_{j \in J} \int_{y \in E_{j}} m_{r}(\mathrm{~d} y)\left(\left(P^{\prime} P^{k}\right)\left(y, K^{c}\right)-\left(P^{\prime} P^{k}\right)\left(x_{j}, K^{c}\right)\right) \\
\leq & \sum_{j \in J}\left[m_{r}\left(E_{j}\right)\left(P^{\prime} P^{k}\right)\left(x_{j}, K^{c}\right)\right]+\tilde{\delta} \tilde{l}_{P, P^{\prime}} \\
\leq & \tilde{\delta}+\tilde{\delta} \tilde{l}_{P, P^{\prime}},
\end{aligned}
$$

where the next to last inequality follows from Assumption 1.7 (2). Finally, $\forall n \geq k_{0}+1$

$$
\begin{aligned}
\eta_{n+1}\left(K^{c}\right) & \leq \sum_{k=0}^{k_{0}}\left[\epsilon(1-\epsilon)^{k} m_{n-k} P^{\prime} P^{k}\left(K^{c}\right)\right]+\tilde{\delta} \\
& \leq \sum_{k=0}^{k_{0}}\left[\epsilon(1-\epsilon)^{k}\left(\tilde{\delta}+\tilde{\delta} \tilde{l}_{P, P^{\prime}}\right)\right]+\tilde{\delta}
\end{aligned}
$$


The first statement follows. To prove the second statement, we begin by defining the (random )operator $S^{N} \circ P$ acting on probability measures on $\mathbb{R}^{d}: \mu\left(S^{N} \circ P\right)=\left(S^{N}(\mu)\right) P$. We have

$$
\tilde{\eta}_{n+1}^{N}=\sum_{k=0}^{n}\left[\epsilon(1-\epsilon)^{k} \tilde{m}_{n-k}^{N} P^{\prime}\left(S^{N} \circ P\right)^{k}\right]+(1-\epsilon)^{n+1} \eta_{0}\left(S^{N} \circ P\right)^{n+1} .
$$

We take the same $\tilde{\delta}, x_{j}, K_{j}, \ldots$ as above and we notice that $\forall k, j$,

$$
\mathbb{E}\left(\delta_{x_{j}} P^{\prime}\left(S^{N} \circ P\right)^{k}\left(K_{j}^{c}\right)\right)=\delta_{x_{j}} P^{\prime} P^{k}\left(K_{j}^{c}\right) .
$$

From this point, the proof is the same as for the first statement.

Acknowledgements. AB has been supported in part by the National Science Foundation (DMS-1004418, DMS-1016441), the Army Research Office (W911NF-0-1-0080, W911NF-10-1-0158) and the US-Israel Binational Science Foundation (2008466). Part of this work was done when AB was visiting Université de Nice-Sophia Antipolis and Université de Bordeaux I and when SR was visiting University of North Carolina at Chapel Hill. Hospitality of these universities is gratefully acknowledged.

\section{REFERENCES}

[1] N. Bartoli and P. Del Moral, Simulation \& Algorithmes Stochastiques. Cépaduès éditions (2001).

[2] L. Bertini, G. Giacomin and K. Pakdaman, Dynamical aspects of mean field plane rotators and the Kuramoto model. J. Stat. Phys. 138 (2010) 270-290.

[3] F. Caron, P. Del Moral, A. Doucet and M. Pace, Particle approximations of a class of branching distribution arising in multi-target tracking. SIAM J. Contr. Optim. 49 (2011) 1766-1792.

[4] F. Caron, P. Del Moral, M. Pace and B.-N. Vo, On the stability and the approximation of branching distribution flows, with applications to nonlinear multiple target filtering. Stoch. Anal. Appl. 29 (2011) 951-997.

[5] J. Carrillo, R. McCann and C. Villani, Kinetic equilibration rates for granular media and related equations: entropy dissipation and mass transportation estimates. Rev. Mat. Iberoamericana 19 (2003) 971-1018.

[6] J. Carrillo, R. McCann and C. Villani, Contractions in the 2-Wasserstein length space and thermalization of granular media. Arch. Ration. Mech. Anal. 179 (2006) 217-263.

[7] P. Cattiaux, A. Guillin and F. Malrieu, Probabilistic approach for granular media equations in the non-uniformly convex case. Probab. Theory Relat. Fields 140 (2008) 19-40.

[8] D. Dawson, Critical dynamics and fluctuations for a mean-field model of cooperative behavior. J. Statist. Phys. 31 (1983) 29-85.

[9] R.L. Dobrushin, Central limit theorem for nonstationary Markov chains I. Theory Probab. Appl. 1 (1956) 65-80.

[10] R.L. Dobrushin, Central limit theorem for nonstationary Markov chains II. Theory Probab. Appl. 1 (1956) $329-383$.

[11] A. Friedman and J.I. Tello, Stability of solutions of chemotaxis equations in reinforced random walks. J. Math. Anal. Appl. 272 (2002) 138-163.

[12] J. Gartner, On the McKean-Vlasov limit for interacting diffusions. Math. Nachr. 137 (1988) 197-248.

[13] K. Giesecke, K. Spiliopoulos and R. Sowers, Default clustering in large portfolios: typical events. Ann. Appl. Probab. 23 (2013) $348-385$.

[14] C. Graham and P. Robert, Interacting multi-class transmissions in large stochastic networks. Ann. Appl. Probab. 19 (2009) $2334-2361$.

[15] C.l Graham, J. Gomez-Serrano and J. Yves Le Boudec, The bounded confidence model of opinion dynamics (2010) arxiv.org/pdf/1006.3798.

[16] B. Latané and A. Nowak, Self-organizing social systems: Necessary and sufficient conditions for the emergence of clustering, consolidation, and continuing diversity. Prog. Commun. Sci. (1997) 43-74.

[17] F. Malrieu, Convergence to equilibrium for granular media equations and their Euler schemes. Ann. Appl. Probab. 13 (2003) $540-560$.

[18] F. Schweitzer, Brownian agents and active particles. Springer Series in Synergetics. Collective dynamics in the natural and social sciences, With a foreword by J. Doyne Farmer. Springer-Verlag, Berlin (2003).

[19] A. Stevens, Trail following and aggregation of myxobacteria. J. Biol. Syst. 3 (1995) 1059-1068.

[20] A.-S. Sznitman, Topics in propagation of chaos, in École d'Été de Probabilités de Saint-Flour XIX-1989, vol. 1464 of Lect. Notes Math. Springer, Berlin (1991) 165-251. 